PROC IMECHE-PART N- JOURNAL OF NANOMATERIALS,

NANOENGINEERING AND NANOSYSTEMS

PUBLISHERS: SAGE

ACCEPTED JUNE $2^{N D} 2017$

elSSN: 23977922 | ISSN: 23977914

\title{
FINITE ELEMENT COMPUTATION OF MAGNETOHYDRODYNAMIC NANOFLUID CONVECTION FROM AN OSCILLATING INCLINED PLATE WITH RADIATIVE FLUX, HEAT SOURCE AND VARIABLE TEMPERATURE EFFECTS
}

\author{
Thirupathi Thumma $^{1^{*}}$, O. Anwar Beg ${ }^{2}$ and Siva Reddy Sheri ${ }^{3}$ \\ ${ }^{1}$ Department of Mathematics, B V Raju Institute of Technology, Medak, 502313, Telangana, India. \\ ${ }^{2}$ Fluid Mechanics, Spray Research Group, Petroleum/Gas Engineering Division, Newton Bldg., \\ University of Salford, Manchester, M54WT, UK. \\ ${ }^{3}$ Department of Mathematics, Gandhi Institute of Technology and Management University, \\ Hyderabad Campus, India.
}

*Corresponding Author: Email- thirupathi.thumma@gmail.com

\begin{abstract}
The present work describes finite element computations for radiative magnetohydrodynamic convective Newtonian nanofluid flow from an oscillating inclined porous plate with variable temperature. Heat source/sink and buoyancy effects are included in the mathematical model. The problem is formulated by employing Tiwari-Das nanofluid model and two water - based nanofluids with spherical shaped metal nano particles as copper and alumina are considered. The Brinkman and Maxwell-Garnetts models are used for the dynamic viscosity and effective thermal conductivity of the nanofluids respectively. An algebraic flux model, the Rosseland diffusion approximation is adopted to simulate thermal radiative flux effects. The dimensionless, coupled governing partial differential equations are numerically solved via the finite element method with weak variational formulation by imposing initial and boundary conditions with a weighted residual scheme. A grid independence study is also conducted. The finite element solutions are reduced to known previous solutions in some limiting cases of the present investigation and are found to be in good agreement with published work. This investigation is relevant to electromagnetic nanomaterial manufacturing processes operating at high temperatures where radiation heat transfer is significant.
\end{abstract}

Keywords: Oscillatory Inclined Surface, Magneto-Nanofluid Dynamics, Thermal Convection, Radiation, Heat Source/Sink, Variable Temperature, Finite Element Method, Nano-Materials Fabrication.

\section{Introduction}

Nanofluids are fabricated by suspending nanoparticles $\left(\mathrm{Cu}, \mathrm{Al}, \mathrm{Al}_{2} \mathrm{O}_{3}, \mathrm{TiO}_{2}, \mathrm{SiC}, \mathrm{SiN}\right.$, $A l N)$ with average sizes less than $100 \mathrm{~nm}$ in conventional heat transfer fluids $\left(\mathrm{H}_{2} \mathrm{O}\right.$, $\mathrm{C}_{2} \mathrm{H}_{6} \mathrm{O}_{2}$ ) as well as other base fluids like mineral oil, engine oil, and poor heat transfer fluids. Nanofluids emerged following pioneering investigations into a new class of technologies and coolants at Argonne National Laboratory in the United States of America in the 1990s, as 
elaborated by (Choi [1], Choi et al. [2]). Nanofluids describe heat transfer fluids that exhibit thermal properties superior to those of their host fluids. The study of particulate suspensions has remarkable applications which includes high power X-rays, optical devices, material synthesis, lasers and biomedical science to name a few. A comprehensive review on the research progress on the natural convective heat transfer characteristics of nanofluids for both single phase and two-phase models in different types of enclosures is reported by Haddad et al. [3]. Generally two models are popular for simulating nanofluids, namely the Buongiorno model [4] (which highlights the contribution of Brownian diffusion and thermophoresis for heat transfer enhancement) and the Tiwari and Das formulation [5] (which features a nanoparticle volume fraction). In the former [4] a separate mass (species) diffusion equation in addition to momentum and heat conservation equations is required whereas in the latter [5] only momentum and energy conservations equations are considered. We employ the latter in the present study.

The magnetohydrodynamic (MHD) flow and heat transfer in both purely fluid and porous media finds significant applications in materials processing (e.g. liquid metals, polymers, lubricants). Examples include rapid thermal processing [6], liquid flow control [7], induction heating in contactless stirring and surface modification [8], transverse magnetic fieldcontrolled segregation during directional solidification [9], thermal transport in magnetized liquid metal boundary layers [10] and buoyancy control of slurry materials in porous materials via magnetic fields $[10,11]$. These works have explored many complex hydromagnetic phenomena including magnetic induction, Lorentzian body forces, magnetic levitation, AC and DC magnetic field and thermal physics. In recent years the emergence of nanomaterials doped with magnetic nano-particles has also rekindled interest in electromagnetic flows of nanofluids. In parallel with experimental studies of magnetic nanofluid synthesis $[12,13]$, computational simulation has also drawn significant attention in engineering sciences, in particular where multiple physico-chemical effects arise. These studies span materials operations, biomedical systems and energy sciences and have employed many sophisticated analytical and numerical approaches. Mahanthesh et al. [14] investigated heat and mass transfer effects on the mixed convective flow of chemically reacting nanofluid from a moving or stationary vertical plate, deriving closed form solutions and considering dual buoyancy i.e. both positive and negative Grashof number cases. Akbar et al. [15] utilized Mathematica integration routines to derive solutions for brick, cylinder and disk-shaped nanoparticles in heat transfer and peristaltic propulsion of magnetic nanofluids in cylindrical conduits. Thiagarajan and Selvaraj [16] studied numerically nonlinear hydromagnetic stagnation point flow and heat transfer of diamond, platinum-mineral oil based nanofluid with viscous dissipation from a horizontal surface. Das and Jana [17] employed a Laplace Transform (LT) technique to explore the free convection magnetonanofluid flow and radiative heat transfer from both stationary and translating vertical sheet cases. Siva Reddy and Thirupathi [18] investigated heat and mass transfer effects on natural convection flow in the presence of volume fraction for copper-water nanofluid by employing the finite element method. Ferdows et al. [19] used an explicit finite difference method to investigate combined thermal radiation and transverse magnetic field effects on unsteady magneto-convection from an extensing nano-polymer sheet with wall mass flux effects. Gireesha et al. [20] used a Runge-Kutta-Fehlberg method with shooting technique to study the influence of suspended nanoparticles on three dimensional MHD flow, heat and mass transfer of a radiating Eyring-Powel fluid over a stretching sheet, observing the strong effect of transverse magnetic field on flow characteristics. Uddin et al. [21] used a high penalty finite element algorithm to examine magnetic field and wall slip effects on both stretching and contracting electrically-conducting nanofluid sheet flows in Darcian porous media. 
Sheikholeslami et al. [22] used the Lattice Boltzmann method to compute buoyancy, radiative flux and magnetic field effects on Aluminium oxide water nanofluid transport in porous materials. Uddin et al. [23] employed both shooting methods and Chebyshev spectral collocation techniques to compute Hartmann number (magnetic parameter) effects on slip magnetic nanofluid flows from both stretching and shrinking sheet flows.

The above studies were generally restricted to vertical or horizontal external boundary layer flows in magnetic nanofluid flows. However in certain operations, the use of gravity may be exploited via inclination of the manufacturing surface. This allows for example a scaling of the thermal buoyancy force with inclination of the plate. Applications in this regard include electroplating, enrobing and surface deposition of magnetic nano-particles [24]. Many studies have considered inclination effects for different magnetic material models or heat transfer effects but ignored nanoscale effects. Other investigations have considered nanofluid transport from inclined surfaces but have neglected magnetohydrodynamics. Examples of the former include micropolar magnetic flow from a titled plane, as studied by Beg et al. [25] using network simulation, Ramadan and Chamkha [26] who studied magnetic inclined dusty flows, Subba Rao et al. [27] who examined Casson viscoplastic slip boundary layers from inclined surfaces and Usha and Uma [28] who simulated viscoelastic wavy flows from inclined surfaces. Further studies of non-nanofluid transport from inclined planes include Beg et al. [29] for unsteady radiative flows, Sui et al. [30] for pseudo plastic and dilatant mixed convection flows and Noor et al. [31] for particle deposition in radiative magnetic inclined plate convection. Regarding heat transfer in nanofluid flows from inclined surfaces, representative studies (which are few) include Rana et al. [32] who also considered porous media and Abed [33] who studied experimentally the thermos-gravitational convection in an inclined layer of nanofluid heated from below. Relatively sparse investigations of external magnetic nanofluid boundary layer flows from inclined surfaces have been reported. Rana and Beg [34] conducted one of the first investigations into inclination effects on magnetohydrodynamic mixed convection flow along an inclined porous sheet, presenting finite element solutions and noting in detail the influence of plate tilt angle, thermal conductivity and nanoparticle geometric effects on heat and mass transfer rates. Very recently Dhanai et al. [35] studied multiple slip effects on hydromagnetic nanofluid convection from an inclined cylinder using numerical quadrature methods. They emphasized the strong influence of inclination angle, buoyancy and hydrodynamic and thermal slip on wall shear stress and heat transfer rates.

In view of the above studies, it is evident, to the best of authors knowledge, that no attempts far been instigated with regard to studying oscillating inclined magnetohydrodynamic nanofluid transport with thermal radiative effects. In the present work, a detailed finite element numerical simulation is therefore presented to study radiative magnetohydrodynamic convective Newtonian nanofluid flow from an oscillatory inclined porous surface with variable temperature. Furthermore in the present model the supplementary effects of heat source/sink, buoyancy and porous media body force are also considered. This extends the existing simulations of magnetic nanofluid flows by considering simultaneously the supplementary effects of heat source/sink, porous media and also inclination of the manufacturing surface, which arise in numerous high-temperature process engineering operations. These effects have been considered independently in previous works [36-40]. A robust finite element method with Galerkin weighted residual scheme is utilized in the current work. The numerical values for governing physical parameters on velocity and temperature distributions are depicted graphically. To ascertain the correctness of the numerical results the skin friction, Nusselt number values with in the flow regime are 
computed and documented in tabular form. We reported the effects of appropriate physical parameters on velocity and temperature distributions for two water based nanofluids $\mathrm{Cu}-\mathrm{H}_{2} \mathrm{O}$ and $\mathrm{Al}_{2} \mathrm{O}_{3}-\mathrm{H}_{2} \mathrm{O}$ in the cases of sinusoidal and co-sinusoidal oscillations of an inclined porous plate. Furthermore, the formulation of the problem and method of solution are presented in sections 2 and 3 respectively. Results and discussions are reported in section 4 finally important results are summarized in section 5 . The current study constitutes a significant extension to the existing simulations which are relevant to high temperature radiative transient magnetic nano-materials processing [41].

\section{Mathematical magnetized nanofluid model}

Consider the unsteady magnetohydrodynamic free convection flow of an electrically conducting, viscous, incompressible water based nanofluids past a semi-infinite permeable oscillatory inclined surface with an acute angle $\left(0^{0} \leq \alpha \leq 90^{\circ}\right)$ to the vertical, embedded in uniform porous medium in the presence of thermal buoyancy and constant heat source/sink effects. The temperature is high enough to invoke significant thermal radiation effects. The mathematical model of the problem is formulated by choosing the cartesian coordinate system $\left(x^{*}, y^{*}\right)$ as shown in Figure 1 in such a way that the boundary layer grows along the $x^{*}$-axis i.e. along the inclined plate direction, $y^{*}$-axis is perpendicular to the plate surface. The inclination angles $0^{0}, 90^{\circ}$ and $0^{\circ}<\alpha<90^{\circ}$ represents the vertical, horizontal and inclined plate scenarios respectively.

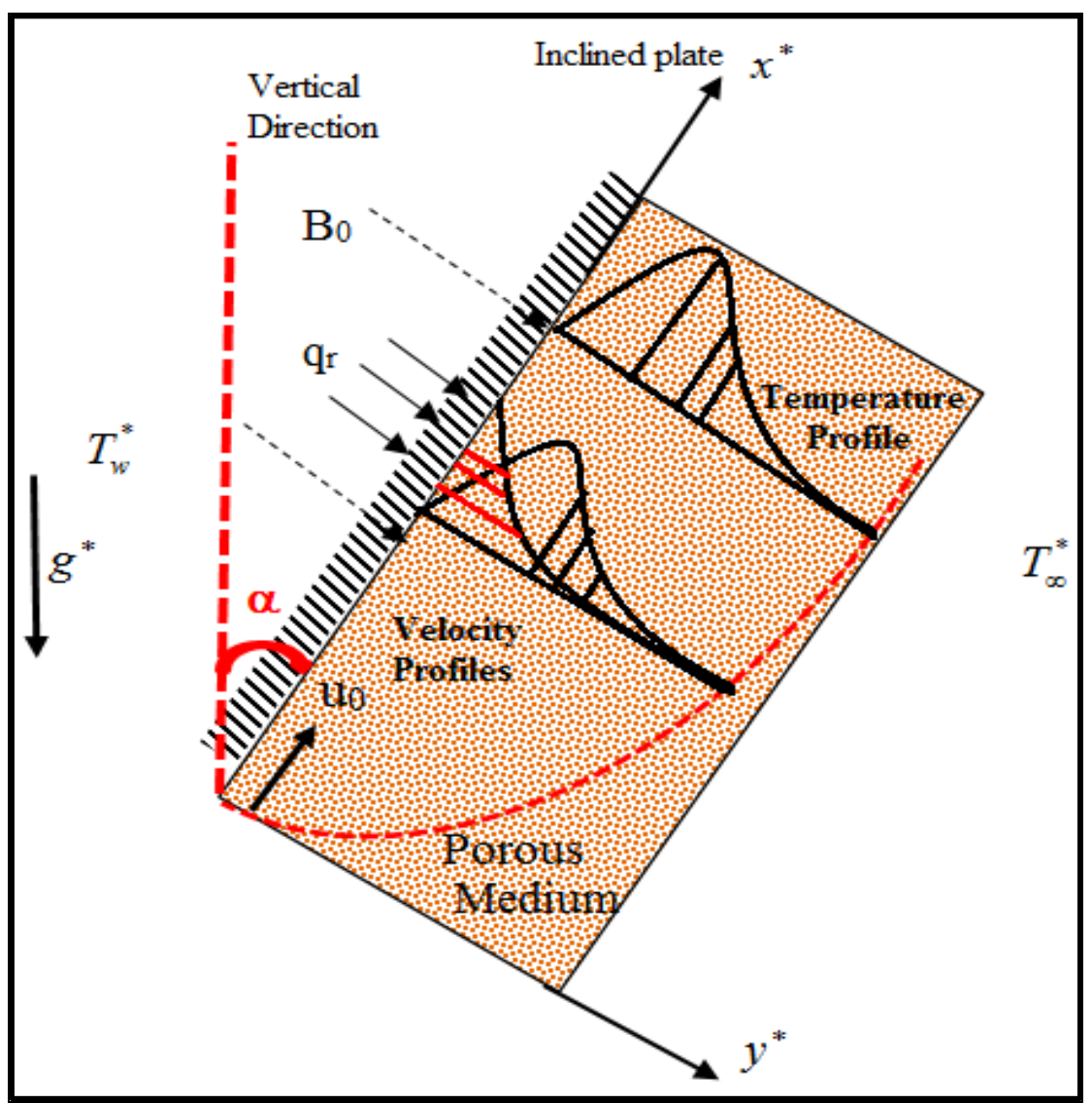

Figure 1 Physical model for nano-materials processing system with co-ordinates.

The following assumptions are implicit in the present model. 
- Initially i.e. at time $t^{*} \leq 0$, both plate and the magnetic nanofluid are at rest with uniform temperature $T_{\infty}^{*}$. It is assumed that flow is driven by the oscillatory movement of the inclined plate, thus, at time $t^{*}>0$ i.e. once the plate starts oscillating in $x^{*}$-axis direction the temperature of the plate is raised linearly with time $t^{*}$ with time dependent velocity $u^{*}\left(0, t^{*}\right)=u_{0}\left\{a e^{i n^{*} t^{*}}+b e^{-i n^{*} t^{*}}\right\}$ where uo is the characteristic velocity, $n^{*}$ is the frequency of oscillations, $t^{*}$ denotes time and $a, b$ are complex constants.

- The surface temperature is assumed to have the constant value $T_{w}^{*}$ which is greater than the ambient temperature value of $T_{\infty}^{*}$.

- The plate (sheet) is electrically non-conducting and the fluid is optically thick, gray, absorbing and emitting but non-scattering [42].

- The radiative heat flux $q_{r}$ is applied normal to the plate surface (i.e. flow direction) and is negligible in the $x^{*}$-axis direction as compared to that in $y^{*}$-axis direction.

- An external magnetic field of uniform strength $B_{0}$ is applied transversely to the direction of fluid flow i.e. along the $y^{*}$-axis. The induced magnetic field generated due to the movement of the fluid and is negligible in comparison with the applied external magnetic field i.e. magnetic Reynolds number is assumed to be small ((Liron and Wilhelm [43])) for metallic liquids and partially ionized fluids.

- There is no applied voltage which implies the absence of an electrical field so that the effects of polarization of the nanofluid are negligible. Furthermore, induced magnetic field is negligible, then the Maxwell's equation $\nabla \times \vec{E}=\frac{-\partial \vec{B}}{\partial t}$ becomes $\nabla \times \vec{E}=0$ which gives rise to $\frac{\partial E_{x}}{\partial t}=0$ i.e. $E_{x}=$ consant everywhere in the flow and this constant is taken to be zero. Therefore, we have $E_{x}=0$.

- Since the plate is infinite in extent, all physical quantities which govern the problem depend on $y^{*}$ and $t^{*}$ only.

- The base fluid and suspended nano particles with uniform shape and size are in thermal equilibrium state and no slip occurs between them.

- High permeability of the porous medium is assumed and the medium s furthermore homogenous, isotropic and fully-saturated with magnetic nanofluid.

Incorporating the above assumptions, the dimensional governing equations (Rajesh et al., [50]) for unsteady free convective-radiative heat and mass transfer flow of an incompressible viscous Newtonian magnetic nanofluid, in the presence of heat generation/absorption under the Boussinesq and boundary layer approximations may be shown to take the form:

$$
\begin{aligned}
& \rho_{n f}\left(\frac{\partial u^{*}}{\partial t^{*}}\right)=\mu_{n f} \frac{\partial^{2} u^{*}}{\partial y^{* 2}}+g(\rho \beta)_{n f}\left(T^{*}-T_{\infty}^{*}\right) \cos (\alpha)-\sigma_{n f} B_{0}{ }^{2} u^{*}-\frac{v_{f} u^{*}}{k} \\
& \frac{\partial T^{*}}{\partial t^{*}}=\alpha_{n f} \frac{\partial^{2} T^{*}}{\partial y^{* 2}}-\frac{1}{\left(\rho C_{P}\right)_{n f}} \frac{\partial q_{r}}{\partial y^{*}}-\frac{Q^{*}}{\left(\rho C_{P}\right)_{n f}}\left(T^{*}-T_{\infty}^{*}\right)
\end{aligned}
$$


Here, $u^{*}$ is velocity component along the $x^{*}$ direction respectively, $T^{*}$ denotes temperature of the nanofluid, $\rho_{n f}=(1-\phi) \rho_{f}+\phi \rho$ is effective density of nanofluid, $\alpha_{n f}=k_{n f} /\left(\rho c_{p}\right)_{n f}$ is thermal diffusivity of the nanofluid, $\left(\rho C_{p}\right)_{n f}=(1-\phi)\left(\rho C_{p}\right)_{f}+\phi\left(\rho C_{p}\right)$ is the heat capacitance of the nanofluid, $(\rho \beta)_{n f}=(1-\phi)(\rho \beta)_{f}+\phi(\rho \beta)$ is the thermal expansion coefficient and $\sigma_{n f}=\sigma_{f}\left(1+3\left(\left(\sigma_{s} / \sigma_{f}\right)-1\right) \phi /\left(\left(\sigma_{s} / \sigma_{f}\right)+2\right)-\left(\left(\sigma_{s} / \sigma_{f}\right)-1\right) \phi\right)$ is electrical conductivity of the nanofluid. Furthermore, $\beta$ is thermal expansion coefficient $\left(\mathrm{K}^{-1}\right), \beta_{f}$ is coefficient of thermal expansion of the fluid $\left(\mathrm{K}^{-1}\right), \beta_{s}$ is coefficient of thermal expansion of the solid $\left(\mathrm{K}^{-1}\right), \rho_{f}$ density of the fluid $\left(\mathrm{kgm}^{-3}\right), \rho_{s}$ is density of the solid friction $\left(\mathrm{kgm}^{-3}\right), \rho_{n f}$ density of the nanofluid $\left(\mathrm{kgm}^{-3}\right), \mu_{n f}$ is dynamic viscosity of the magnetic nanofluid $\left(\mathrm{m}^{2} \mathrm{~s}^{-1}\right)$, $v_{f}$ is kinematic viscosity of the fluid $\left(\mathrm{m}^{2} \mathrm{~s}^{-1}\right), k$ is the permeability of the porous medium $\left(\mathrm{m}^{2}\right), \sigma_{s}$ electrical conductivity of the nanoparticles $\left(\mathrm{Sm}^{-1}\right), \sigma_{f}$ electrical conductivity of the base fluid $\left(\mathrm{Sm}^{-1}\right)$. In equation (1) the last term designates the Darcian porous medium impedance which is valid for low Reynolds numbers. The effective thermal conductivity $K_{n f}$ of the nanofluid for spherical nanoparticles is given by the formulations presented in Hamilton and Cross [44], Kakac and Pramuanjaroenkij [45] and Oztop and Abu-Nada [46] and takes the form $K_{n f}=K_{f}\left[\frac{K_{s}+\left(n_{1}-1\right) K_{f}-\left(n_{1}-1\right) \phi\left(K_{f}-K_{s}\right)}{K_{s}+\left(n_{1}-1\right) K_{f}+\phi\left(K_{f}-K_{s}\right)}\right]$ where $k_{s}$ is thermal conductivity of the solid $\left(\mathrm{Wm}^{-1} \mathrm{~K}^{-1}\right), K_{f}$ is thermal conductivity of the fluid $\left(\mathrm{Wm}^{-1} \mathrm{~K}^{-1}\right), K_{n f}$ is thermal conductivity of the nanofluid $\left(\mathrm{Wm}^{-1} \mathrm{~K}^{-1}\right), \phi$ volume fraction parameter, $n_{1}$ is the empirical shape factor (in particular, $n_{1}=3$ for spherical shaped nanoparticles and $n_{1}=3 / 2$ for cylindrical shaped nanoparticles) for the nanoparticles, the subscripts $f$ is fluid, $s$ is solid, $n f$ is nanofluid. The effective dynamic viscosity of the nanofluid as given by Brinkman [47] is $\mu_{n f}=\frac{\mu_{f}}{(1-\phi)^{2.5}}$ where $\mu_{f}$ dynamic viscosity of the fluid $\left(\mathrm{Nsm}^{-2}\right), \mu_{n f}$ viscosity of the nanofluid $\left(\mathrm{Nsm}^{-2}\right)$. It is worth mentioning that in practical materials processing systems perfect insulation is not feasible. There will always be some fluctuation in the temperature, and to simulate this, the temperature is taken to vary linearly with time. The hydromagnetic flow at an oscillating plate has important application in acoustic streaming problems also. Therefore, the corresponding initial and boundary conditions for velocity distributions (Vajravelu and Rivera [48] and Das et al. [49]) and for the temperature distributions (Rajesh et al. [50]) on the vertical surface and in free stream may be defined as:

$$
\left.\begin{array}{ll}
\forall y^{*} & : \text { for } t^{*} \leq 0 ; u^{*}\left(y^{*}, t^{*}\right)=0, T^{*}\left(y^{*}, t^{*}\right)=T_{\infty}^{*}, \\
\text { At } y^{*}=0 \quad: \text { for } t^{*}>0 ; u^{*}\left(0, t^{*}\right)=u_{0}\left\{a e^{i n^{*} t^{*}}+b e^{-i n^{*} t^{*}}\right\} T^{*}\left(0, t^{*}\right)=T_{\infty}^{*}+\left(T_{w}^{*}-T_{\infty}^{*}\right) A t^{*}, \\
\text { As } y^{*} \rightarrow \infty \quad: \text { for } t^{*}>0 ; u^{*}\left(\infty, t^{*}\right) \rightarrow 0, T^{*}\left(\infty, t^{*}\right) \rightarrow T_{\infty}^{*}
\end{array}\right\}
$$

For an optically-thick fluid, in addition to emission there is also self-absorption and usually the absorption coefficient is wavelength-dependent and large, permitting the implementation of the Rosseland diffusion flux approximation (Brewster [51]). In this way, the net radiative heat flux term can be simplified to:

$q_{r}=\frac{-4 \sigma^{*}}{3 k^{*}} \frac{\partial T^{* 4}}{\partial y^{*}}$

Where $k^{*}\left(m^{-1}\right)$ and $\sigma^{*}\left(5.67 \times 10^{-8} w / m^{2} k^{4}\right)$ are the Rosseland mean absorption coefficient 
and Stefan-Boltzmann constant, respectively. It is assumed that the temperature difference within the flow is sufficiently small such that $T^{* 4}$ may be expressed as a linear function of the temperature by expanding in a Taylor series about free stream temperature $T_{\infty}^{*}$ as

$T^{*^{4}} \cong T_{\infty}^{*^{4}}+4 T_{\infty}^{*^{3}}\left(T^{*}-T_{\infty}^{*}\right)+6 T_{\infty}^{*^{2}}\left(T^{*}-T_{\infty}^{*}\right)^{2}+\ldots$

Neglecting the higher order terms in Equation (5), we have:

$T^{*^{4}} \cong 4 T_{\infty}^{* 3} T^{*}-3 T_{\infty}^{* 4}$

Hence, Equation (4), by virtue of Equation (9), emerges as:

$\frac{\partial q_{r}}{\partial y^{*}}=-\frac{16 \sigma^{*} T_{\infty}^{* 3}}{3 k^{*}} \frac{\partial^{2} T^{*}}{\partial y^{* 2}}$

The analysis may further be simplified by introducing non-dimensional variables. These are defined as follows:

$$
\left.y=\frac{u_{o} y^{*}}{v_{f}}, u=\frac{u^{*}}{u_{o}} t=\frac{u_{o}^{2} t^{*}}{v_{f}}, n_{1}=\frac{v n^{*}}{u_{0}^{2}}, K=\frac{\rho_{f} k u_{o}^{2}}{v_{f}^{2}}, \theta=\frac{T^{*}-T_{\infty}^{*}}{T_{w}^{*}-T_{\infty}^{*}}, A=\frac{u_{0}^{2}}{v_{f}},\right\}
$$

Here $y$ is dimensional $y^{*}$-coordinate, $u$ is dimensional $x^{*}$-direction velocity, $t$ is dimensionless time, $n^{*}$ is the dimensionless oscillation frequency, $t$ is dimensionless time (containing the suction velocity, $u_{0}$ at the plate), $K$ is the dimensionless permeability parameter, $\theta$ is dimensionless temperature function. Substituting expressions for nanofluid properties, the dimensionless variables in equation (8) and the radiative flux expression from equation (7) into Equations (1) and (2) leads to the following non-dimensional conservation equations for momentum and heat:

$$
\begin{aligned}
& \frac{\partial u}{\partial t}=A_{1} \frac{\partial^{2} u}{\partial y^{2}}+A_{2} G r \theta \cos (\alpha)-A_{3}\left(M^{2}+\frac{1}{K}\right) u \\
& \frac{\partial \theta}{\partial t}=A_{5} \frac{\partial^{2} \theta}{\partial y^{2}}-A_{4} Q \theta
\end{aligned}
$$

Where $A_{1}=\frac{1}{(1-\phi)^{2.5} \rho_{n f}}, A_{2}=\frac{(\rho \beta)_{n f}}{(\rho)_{n f}}, A_{3}=\frac{\sigma_{n f}}{\rho_{n f}}, A_{4}=\frac{1}{(\rho C p)_{n f} \operatorname{Pr}}, A_{5}=A_{4}\left(K_{n f}+R\right)$, $M^{2}=\frac{\sigma_{f} B_{o}^{2} v_{f}}{\rho_{f} u_{o}^{2}}$ is the magnetic body force parameter, $R=\frac{16 \sigma^{*} T_{\infty}^{* 3}}{3 k k^{*}}$ is the radiation-conduction parameter, $\operatorname{Pr}=\frac{\left(\rho C_{p}\right)_{f} v_{f}}{k_{f}}$ is the Prandtl number, $G r=\frac{g \beta_{f} v_{f}\left(T_{\infty}^{*}-T_{\infty}^{*}\right)}{u_{0}{ }^{3}}$ is the thermal Grashof number and $Q=\frac{Q^{*} v_{f}^{2}}{k_{f} U_{0}^{2}}$ is the heat source/sink parameter.

The associated dimensionless initial and boundary conditions are:

$$
\left.\begin{array}{lll}
\forall y & : \text { for } t \leq 0 & u(y, t)=0,, \theta(y, t)=0, \\
\text { At } y=0 & : \text { for } t>0 & u(0, t)=\left(a e^{i n_{1} t}+b e^{-i n_{1} t}\right), \theta=t \\
\text { As } y \rightarrow \infty & : \text { for } t>0 & u(\infty, t) \rightarrow 0, \theta(\infty, t) \rightarrow 0
\end{array}\right\}
$$




\section{Finite element method numerical solutions}

The transformed system of non-homogeneous and coupled dimensionless partial differential Equations (9) and (10) under the boundary conditions Equation (11) are solved numerically by using a robust finite element method with a Galerkin weighted residual scheme. This method comprises five fundamental steps, namely discretization of the domain, derivation of the element equations, assembly of element equations, imposition of boundary conditions and finally iterative solution of the assembled equations with for example Chloeski decomposition, Householder's method, Gaussian elimination etc. Further details of the finite element technique are given in Reddy [52] with recent applications in unsteady nanofluid dynamics provided also in [37] and [53]. Mathematical formulations for the present finite element approximations are also provided in the Appendix. Dimensionless velocity $(u)$ and temperature $(\theta)$ are computed at various $y$ values with variation in different parameters e.g. $\mathrm{Gr}, \mathrm{t}, \mathrm{Pr}, \mathrm{K}$ etc. Thermo-physical properties of $\mathrm{H}_{2} \mathrm{O}, \mathrm{Cu}, \mathrm{Ag}, \mathrm{Al}_{2} \mathrm{O}_{3}$ and $\mathrm{TiO}_{2}$-water nanofluids are adopted from (Oztop and Abu-Nada [40]) and documented in Table 1. Furthermore, it is important to calculate the engineering quantities of interest. These include the skin-friction (surface wall shear stress function) which is obtained as $C_{f}=\mu_{n f}\left(\frac{\partial u}{\partial y}\right)_{y=0}$. The rate of the heat transfer at the plate is defined as a Nusselt number and given by $N u=-K_{n f}\left(\frac{\partial \theta}{\partial y}\right)_{y=0}$.

\section{Grid independence study and validation.}

To verify the accuracy of the FEM computations, a grid independence study is conducted. The entire domain is divided into successively sized grids of mesh density $131 \times 131,151 \times 151$ and $171 \times 171$. The boundary conditions for $y$ at $\infty$ are replaced by a sufficiently large value where the velocity and temperature profiles approach zero. The MAPLE-based FEM code is run when $t=0.5$ for different step sizes and very good agreement between the results for all profiles is achieved as presented in Table 2. After many trials for computational flexibility we imposed $y_{\max }=8$ where $y_{\max } \rightarrow \infty$ i.e., external to the momentum and energy boundary layers and we adopted for all the computations, 151 intervals of equal step size 0.053. At each node, two functions are to be evaluated. Therefore following assembly of the finite elements a set of 302 algebraic equations is generated which is solved iteratively. By introducing boundary conditions, the system of equations is solved systematically. The solution is assumed to be converged when the solution difference satisfies the desired accuracy of $10^{-5}$. Excellent convergence for all the results is achieved.

In order to further validate the accuracy of the numerical results obtained through the MAPLE based finite element code, we compared the present results for skin friction and Nusselt number with the results obtained through earlier analytical studies. The current model reduces to that of Narahari and Ishak [54] for uniform velocity motion in the absence of heat source/sink, magnetic field parameter permeability parameter with $\alpha=0^{0}$, showing very good correlation and the comparisons are shown in Table 3. Furthermore, comparisons of the present finite element solutions with Rajesh et al. [50] have also been made in the absence of heat source/sink and permeability parameter with $\alpha=0^{0}$. The comparison is documented in 
Table 4 and Table 5. The comparisons confirms that the present results are indeed valid and in agreement with the published literature. Therefore, these favorable comparisons justify confidence in the finite element code employed which can be used therefore in presenting further results quantitatively and graphically.

Table 1. Thermo-physical properties of water and nanoparticles

\begin{tabular}{|c|c|c|c|c|c|}
\hline Physical properties & $\mathrm{H}_{2} \mathrm{O}$ & $\mathrm{Cu}$ & $\mathrm{Ag}$ & $\mathrm{Al}_{2} \mathrm{O}_{3}$ & $\mathrm{TiO}_{2}$ \\
\hline $\mathrm{Cp}(\mathrm{j} / \mathrm{kg} \mathrm{k})$ & 4179 & 385 & 235 & 765 & 686.2 \\
\hline$\rho(\mathrm{kg} / \mathrm{m} 3)$ & 997.1 & 8933 & 10500 & 3970 & 4250 \\
\hline $\mathrm{K}(\mathrm{W} / \mathrm{m} \mathrm{k})$ & 0.613 & 401 & 429 & 40 & 8.9538 \\
\hline$\beta \times 10-5(1 / \mathrm{k})$ & 21 & 1.67 & 1.89 & 0.85 & 0.9 \\
\hline$\sigma(\mathrm{S} / \mathrm{m})$ & $5.5 \times 10-6$ & $59.6 \times 106$ & $62.1 \times 106$ & $35 \times 106$ & $2.6 \times 106$ \\
\hline
\end{tabular}

Table 2. Grid independence study for different grid sizes when $t=0.4$

\begin{tabular}{|c|c|c|c|c|c|}
\hline \multicolumn{5}{|c|}{$u$ : velocity } & \multicolumn{3}{c|}{$\theta:$ Temperature } \\
\hline \multicolumn{7}{|c|}{ Grid sizes } & 131 & 171 \\
\hline 0 & 151 & 171 & 1 & 1 & 1 \\
\hline 0.1278 & 0 & 0 & 1 & 0.9655 & 0.9655 \\
\hline 0.2354 & 0.1278 & 0.1278 & 0.9655 & 0.9322 & 0.9322 \\
\hline 0.3278 & 0.3278 & 0.3278 & 0.9001 & 0.9001 & 0.9001 \\
\hline 0.4082 & 0.4082 & 0.4082 & 0.8692 & 0.8692 & 0.8692 \\
\hline 0.4789 & 0.4789 & 0.4789 & 0.8393 & 0.8393 & 0.8393 \\
\hline 0.5413 & 0.5413 & 0.5413 & 0.8105 & 0.8105 & 0.8105 \\
\hline 0.5965 & 0.5965 & 0.5965 & 0.7827 & 0.7827 & 0.7827 \\
\hline 0.6453 & 0.6453 & 0.6453 & 0.7558 & 0.7558 & 0.7558 \\
\hline 0.6884 & 0.6884 & 0.6884 & 0.73 & 0.73 & 0.73 \\
\hline
\end{tabular}

Table 3. Comparison of shear stress and Nusselt number for various parameter values when $\phi=0, K \rightarrow \infty, M=0, Q=0$ and $\alpha=0^{0}$

\begin{tabular}{|c|c|c|c|c|c|c|c|}
\hline \multirow{2}{*}{$R$} & \multirow{2}{*}{$G r$} & \multirow{2}{*}{$\operatorname{Pr}$} & \multirow{2}{*}{$t$} & \multicolumn{2}{|c|}{ Narahari et al. [54] } & \multicolumn{2}{c|}{ Present results } \\
\cline { 4 - 7 } & & & $\tau$ & $N u$ & $\tau$ & $N u$ \\
\hline 1 & & & & 2.1509 & 1.4213 & 2.1508868 & 1.4212927 \\
\hline 10 & & & & 3.1052 & 1.8559 & 3.1051774 & 1.8558916 \\
\hline 100 & & & & 3.1565 & 1.9415 & 3.1565359 & 1.9414623 \\
\hline & 2 & & & 1.9990 & 1.8559 & 1.9990107 & 1.8558916 \\
\hline & 5 & & & 3.1052 & 1.8559 & 3.1051774 & 1.8558916 \\
\hline & 10 & & & 4.9488 & 1.8559 & 4.9487885 & 1.8558916 \\
\hline & & 0.71 & & 3.1052 & 1.8559 & 3.1051774 & 1.8558916 \\
\hline & & 7.1 & & 2.6727 & 5.1946 & 2.6727107 & 5.1946346 \\
\hline & & 8.09 & & 2.6169 & 5.5278 & 2.6168558 & 5.5278125 \\
\hline & & & 0.2 & 3.1052 & 1.8559 & 3.1051774 & 1.8558916 \\
\hline
\end{tabular}




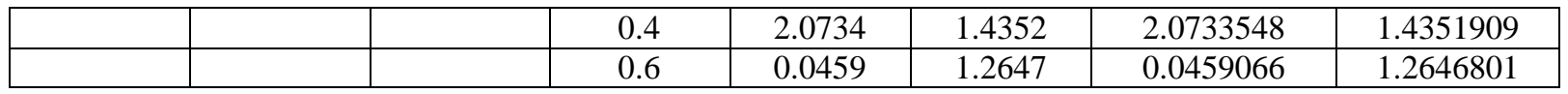

Table 4. Comparison of shear stress for various parameter values when $K \rightarrow \infty, Q=0$ and $\alpha=0^{0}$

\begin{tabular}{|c|c|c|c|c|c|c|c|c|}
\hline \multirow{2}{*}{$G r$} & \multirow{2}{*}{$\phi$} & \multirow{2}{*}{$M$} & \multirow{2}{*}{$a$} & \multirow{2}{*}{$F$} & \multicolumn{2}{|c|}{ Rajesh et al. [50] } & \multicolumn{2}{|c|}{ Present Results } \\
\hline & & & & & $\mathrm{Cu}-\mathrm{H}_{2} \mathrm{O}$ & $\mathrm{Al}_{2} \mathrm{O}_{3}-\mathrm{H}_{2} \mathrm{O}$ & $\mathrm{Cu}-\mathrm{H}_{2} \mathrm{O}$ & $\mathrm{Al}_{2} \mathrm{O}_{3}-\mathrm{H}_{2} \mathrm{O}$ \\
\hline 2 & & & & & -2.6910 & -2.6484 & -2.6910349 & -2.6483594 \\
\hline 5 & & & & & -2.4577 & -2.4158 & -2.4576749 & -2.4157639 \\
\hline 8 & & & & & -2.2243 & -2.1832 & -2.2243150 & -2.1831683 \\
\hline 11 & & & & & -1.9910 & -1.9506 & -1.9909550 & -1.9505727 \\
\hline & 0 & & & & -2.2562 & -2.2562 & -2.2561667 & -2.2561667 \\
\hline & 0.01 & & & & -2.3029 & -2.2944 & -2.3028505 & -2.2943571 \\
\hline & 0.02 & & & & -2.3521 & -2.3337 & -2.3520694 & -2.3336687 \\
\hline & 0.03 & & & & -2.4037 & -2.3741 & -2.4037103 & -2.3741282 \\
\hline & & 1 & & & -1.5597 & -1.4530 & -1.5597226 & -1.4530233 \\
\hline & & 4 & & & -2.4577 & -2.4158 & -2.4576749 & -2.4157639 \\
\hline & & 7 & & & -3.2717 & -3.2653 & -3.2717494 & -3.2652517 \\
\hline & & 10 & & & -3.9859 & -3.9901 & -3.9859091 & -3.9901331 \\
\hline & & & 0.1 & & -1.8205 & -1.7984 & -1.8205040 & -1.7983556 \\
\hline & & & 0.3 & & -2.1201 & -2.0889 & -2.1200767 & -2.0888860 \\
\hline & & & 0.5 & & -2.4577 & -2.4158 & -2.4576749 & -2.4157639 \\
\hline & & & 0.7 & & -2.8379 & -2.7833 & -2.8378894 & -2.7833319 \\
\hline & & & & 1 & -2.4059 & -2.3629 & -2.4058809 & -2.3629021 \\
\hline & & & & 3 & -2.4577 & -2.4158 & -2.4576749 & -2.4157639 \\
\hline & & & & 5 & -2.4713 & -2.4296 & -2.4712788 & -2.4296169 \\
\hline & & & & 7 & -2.4776 & -2.4360 & -2.4775968 & -2.4360465 \\
\hline
\end{tabular}

Table 5. Comparison of Nusselt number for various parameter values when $K \rightarrow \infty, Q=0$ and $\alpha=0^{0}$

\begin{tabular}{|c|c|l|l|l|c|}
\hline \multirow{2}{*}{$\phi$} & \multirow{2}{*}{$F$} & \multicolumn{2}{|c|}{ Rajesh et al. } & \multicolumn{2}{c|}{ Present Results } \\
\cline { 3 - 6 } & & \multicolumn{1}{|c|}{$\mathrm{Cu}-\mathrm{H}_{2} \mathrm{O}$} & \multicolumn{1}{|c|}{$\mathrm{Al}_{2} \mathrm{O}_{3}-\mathrm{H}_{2} \mathrm{O}$} & \multicolumn{1}{c|}{$\mathrm{Cu}-\mathrm{H}_{2} \mathrm{O}$} & $\mathrm{Al}_{2} \mathrm{O}_{3}-\mathrm{H}_{2} \mathrm{O}$ \\
\hline 0 & & 1.6530 & 1.6530 & 1.6530481 & 1.6530481 \\
\hline 0.01 & & 1.6839 & 1.6818 & 1.6839304 & 1.6818136 \\
\hline 0.02 & & 1.7149 & 1.7106 & 1.7149165 & 1.7106400 \\
\hline 0.03 & & 1.7460 & 1.7395 & 1.7460161 & 1.7395357 \\
\hline & $\mathbf{1}$ & 1.4199 & 1.4121 & 1.4199355 & 1.4121112 \\
\hline & $\mathbf{3}$ & 1.7772 & 1.7685 & 1.7772389 & 1.7685088 \\
\hline & $\mathbf{5}$ & 1.8874 & 1.8785 & 1.8873902 & 1.8785184 \\
\hline & $\mathbf{7}$ & 1.9413 & 1.9324 & 1.9413025 & 1.9323874 \\
\hline
\end{tabular}

Table 6. Effects of different values of $\operatorname{Pr}$ and $t$ on Skin friction for different nanofluids.

\begin{tabular}{|c|c|c|c|c|c|}
\hline $\mathrm{Pr}$ & $t$ & $\mathrm{Cu}-\mathrm{H}_{2} \mathrm{O}$ & $\mathrm{Al}_{2} \mathrm{O}_{3}-\mathrm{H}_{2} \mathrm{O}$ & $\mathrm{Ag}-\mathrm{H}_{2} \mathrm{O}$ & $\mathrm{TiO}_{2}-\mathrm{H}_{2} \mathrm{O}$ \\
\hline 11.2 & & -2.5228378 & -2.4817280 & -2.5353696 & -2.4850441 \\
\hline 9.45 & & -2.5046025 & -2.4633093 & -2.5171176 & -2.4666723 \\
\hline 8.09 & & -2.4875563 & -2.4460629 & -2.5000675 & -2.4494682 \\
\hline 7.01 & & -2.4715622 & -2.4298559 & -2.4840801 & -2.4332995 \\
\hline & 0.2 & -2.3247760 & -2.2153331 & -2.3585189 & -2.2218857 \\
\hline & 0.5 & -2.4576749 & -2.4157639 & -2.4702066 & -2.4192397 \\
\hline & 0.8 & -2.6484603 & -2.6093758 & -2.6575331 & -2.6139139 \\
\hline & 1 & -2.7442879 & -2.7040844 & -2.7522725 & -2.7094712 \\
\hline
\end{tabular}


Table 7. Effects of different values of Pr and $t$ on Nusselt number for different nanofluids.

\begin{tabular}{|c|c|c|c|c|c|}
\hline $\mathrm{Pr}$ & $t$ & $\mathrm{Cu}-\mathrm{H}_{2} \mathrm{O}$ & $\mathrm{Al}_{2} \mathrm{O}_{3}-\mathrm{H}_{2} \mathrm{O}$ & $\mathrm{Ag}-\mathrm{H}_{2} \mathrm{O}$ & $\mathrm{TiO}_{2}-\mathrm{H}_{2} \mathrm{O}$ \\
\hline 11.2 & & 2.3886856 & 2.3769518 & 2.3774926 & 2.3515405 \\
\hline 9.45 & & 2.1941478 & 2.1833697 & 2.1838664 & 2.1600279 \\
\hline 8.09 & & 2.0301318 & 2.0201594 & 2.0206189 & 1.9985624 \\
\hline 7.01 & & 1.8897702 & 1.8804872 & 1.8809150 & 1.8603834 \\
\hline & 0.2 & 1.1240246 & 1.1185032 & 1.1187576 & 1.1065455 \\
\hline & 0.5 & 1.7772389 & 1.7685088 & 1.7689111 & 1.7496021 \\
\hline & 0.8 & 2.2480492 & 2.2370063 & 2.2375152 & 2.2130911 \\
\hline & 1 & 2.5133954 & 2.5010491 & 2.5016181 & 2.4743110 \\
\hline
\end{tabular}

\section{Results and discussion}

Extensive numerical results have been obtained for the influence of the emerging nondimensional governing parameters, namely $\alpha, G r, Q, K, M^{2}, n t, \operatorname{Pr}, R$, $t$ and $\phi$ on the nanofluid velocity and temperature distributions for two nanofluids $\mathrm{Cu}-\mathrm{H}_{2} \mathrm{O}$ and $\mathrm{Al}_{2} \mathrm{O}_{3}-\mathrm{H}_{2} \mathrm{O}$. These are visualized graphically in Figures 2 to 15 . The plate oscillates with $\cos (n t)$ and $\sin (n t)$ according to values prescribed as follows for the parameters ' $\mathrm{a}$ ' and ' $\mathrm{b}$ ' as $a=b=1 / 2$ (regarded as case I) and $a=1 / 2 i$ and $b=-1 / 2 i$ (regarded as case II) respectively. In this paper we present the results for the above physical parameters with the following default values prescribed $n=10, t=0.1$ and $K=0.5$. We also adopted the general default values for the other parameters in the finite element computation as $\alpha=45^{\circ}$, $G r=5, Q=1.0, K=0.5 M^{2}=1.0, n t=\pi / 2, \operatorname{Pr}=6.2, R=0.5$ and $\phi=0.1$ unless otherwise stated.

The value of Prandtl number selected is representative of water-based nanofluids. Skin friction and Nusselt number distributions for four nano-particle cases i.e. $\mathrm{Cu}-\mathrm{H}_{2} \mathrm{O}$, $\mathrm{Al}_{2} \mathrm{O}_{3}-\mathrm{H}_{2} \mathrm{O} \mathrm{Ag}-\mathrm{H}_{2} \mathrm{O}$ and $\mathrm{TiO}_{2}-\mathrm{H}_{2} \mathrm{O}$ nanofluids are tabulated in Table 6 and Table 7. Table 6 shows that with increasing Prandtl number the magnitudes of skin friction i.e. shear stress at the plate surface, are generally increased. Skin friction is invariably lowest (i.e. most negative) for $\mathrm{Ag}-\mathrm{H}_{2} \mathrm{O}$ nanofluid, closely followed by $\mathrm{Cu}-\mathrm{H}_{2} \mathrm{O}$ then $\mathrm{TiO}_{2}-\mathrm{H}_{2} \mathrm{O}$ and finally $\mathrm{Al}_{2} \mathrm{O}_{3}-\mathrm{H}_{2} \mathrm{O}$ nanofluid. With increasing time $(t)$, skin friction is strongly decreased i.e. more negative values are generated for all nanofluids, with again strongest deceleration for $\mathrm{Ag}-\mathrm{H}_{2} \mathrm{O}$ nanofluid and weakest deceleration for the $\mathrm{Al}_{2} \mathrm{O}_{3}-\mathrm{H}_{2} \mathrm{O}$ nanofluid. Table 7 shows that with increasing Prandtl number the magnitudes of Nusselt number are generally increased. Nusselt numbers are highest for $\mathrm{Cu}-\mathrm{H}_{2} \mathrm{O}$ nanofluid, closely followed by $\mathrm{Ag}-\mathrm{H}_{2} \mathrm{O}$, then $\mathrm{Al}_{2} \mathrm{O}_{3}-\mathrm{H}_{2} \mathrm{O}$ and finally lowest for $\mathrm{TiO}_{2}-\mathrm{H}_{2} \mathrm{O}$ and nanofluids. With increasing time $(t)$, magnitudes of Nusselt number are all enhanced for each nanofluid, with again $\mathrm{Cu}-\mathrm{H}_{2} \mathrm{O}$ nanofluid attaining the maximum values and $\mathrm{TiO}_{2}-\mathrm{H}_{2} \mathrm{O}$ corresponding to the minimum values.

In order to present the physical insight of the problem under consideration, solutions for two selected different water-based nanofluids $\mathrm{Cu}-\mathrm{H}_{2} \mathrm{O}$ and $\mathrm{Al}_{2} \mathrm{O}_{3}-\mathrm{H}_{2} \mathrm{O}$ are shown graphically 
for velocity and temperature profiles. Fig. 2 depicts the influence of angle of inclination $(\alpha)$ of the plate (sheet) surface on velocity profiles. It is worth mentioning here that in this plot the thermal boundary layer thickness for $\mathrm{Cu}-\mathrm{H}_{2} \mathrm{O}$ nanofluid is generally greater than the $\mathrm{Al}_{2} \mathrm{O}_{3}-\mathrm{H}_{2} \mathrm{O}$ nanofluid, and is attributable to the greater thermal conductivity of copper compared with alumina. The velocity profiles in case I, starts with the plate velocity and increases to a maximum in the momentum boundary layer and decays to asymptotic value with corresponding boundary conditions. Furthermore, the velocity profiles in case II, starts with the plate velocity and then decreases monotonically to zero satisfying the far field boundary condition.

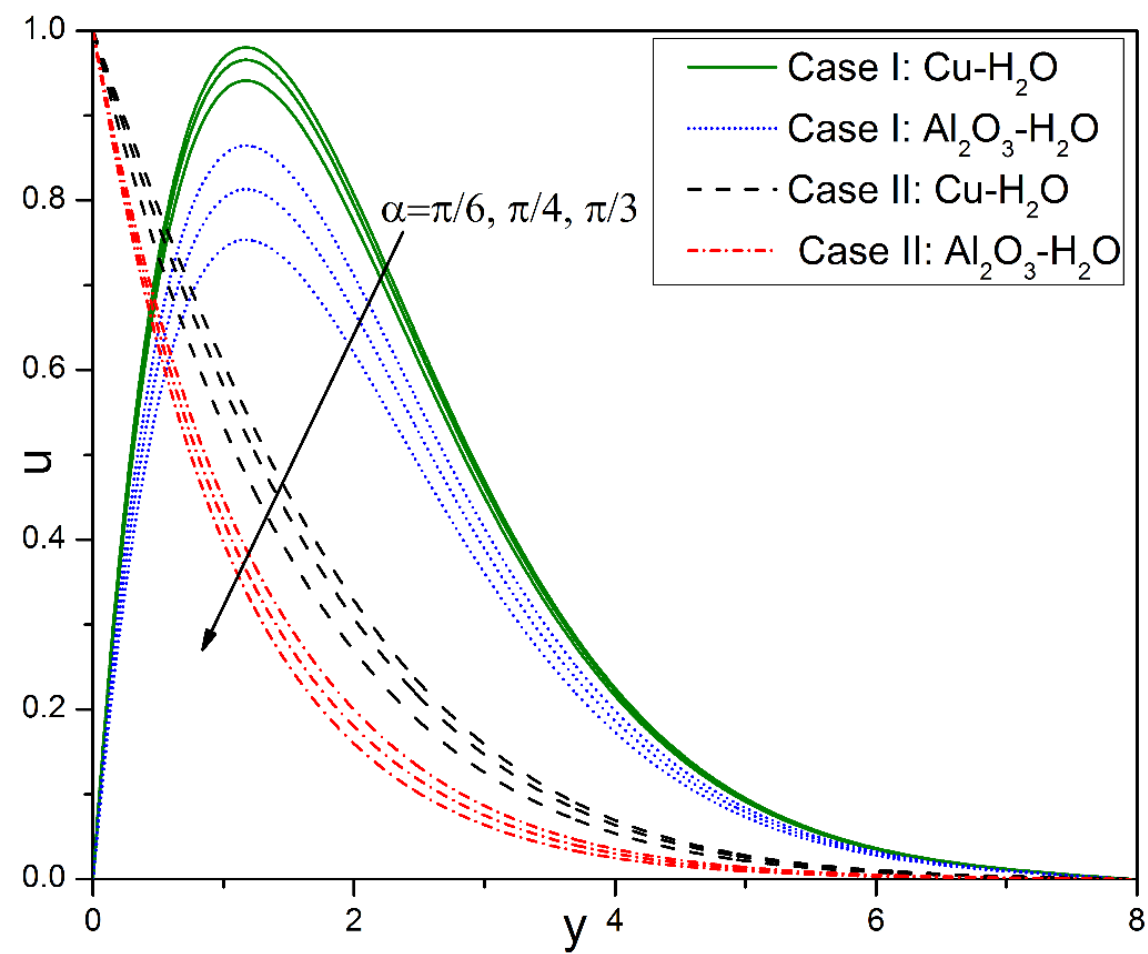

Figure 2: The influence of angle of inclination on velocity profiles. 


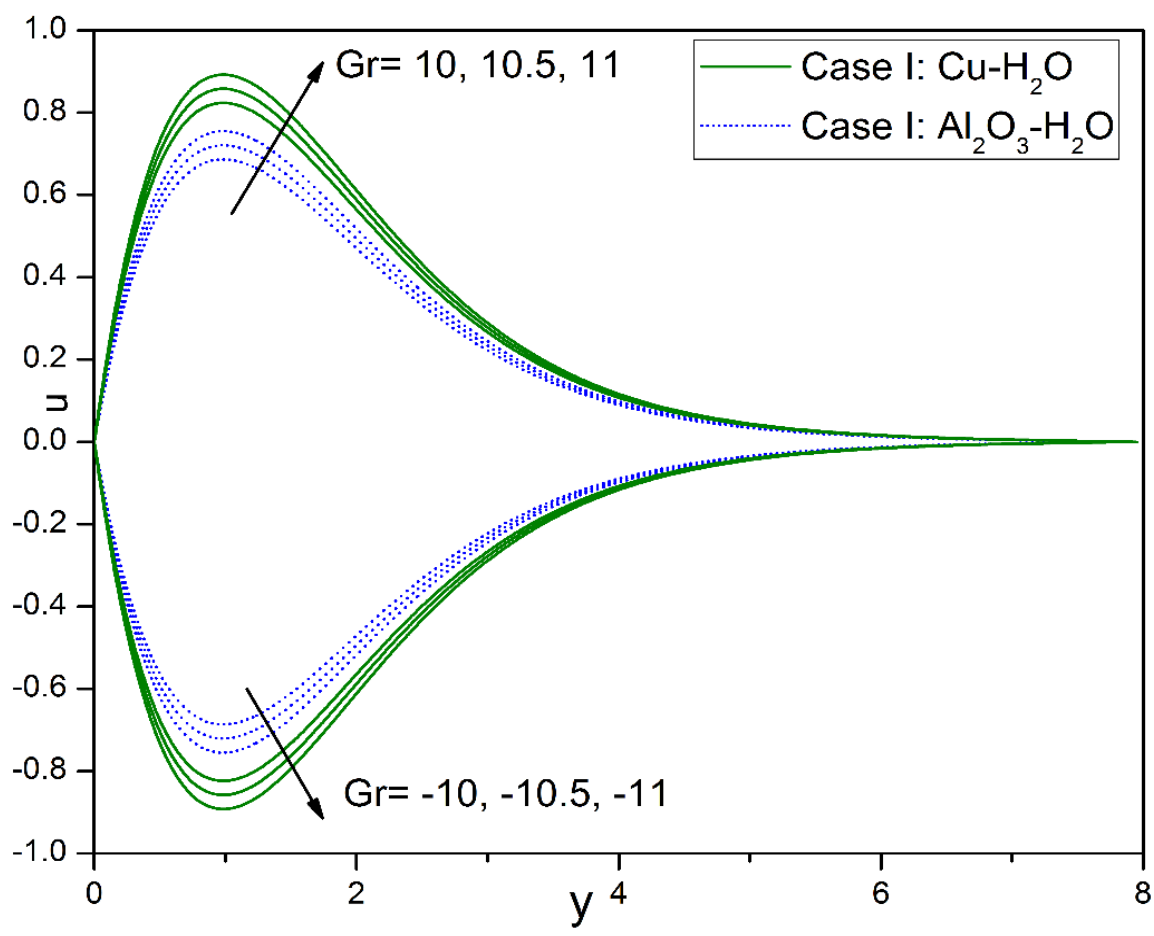

Figure 3: The influence of thermal Grashof Number on velocity profiles.

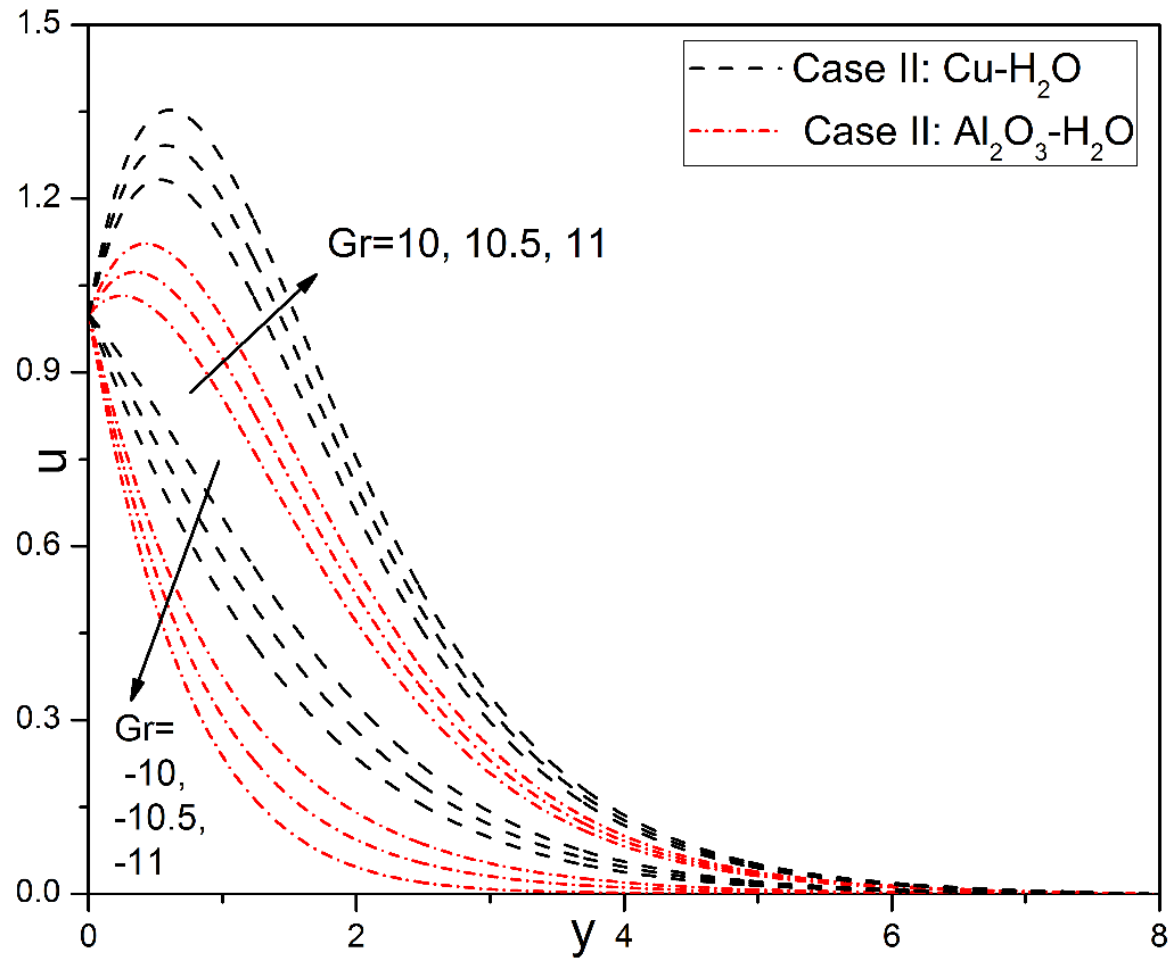

Figure 4: The influence of thermal Grashof Number on velocity profiles. 


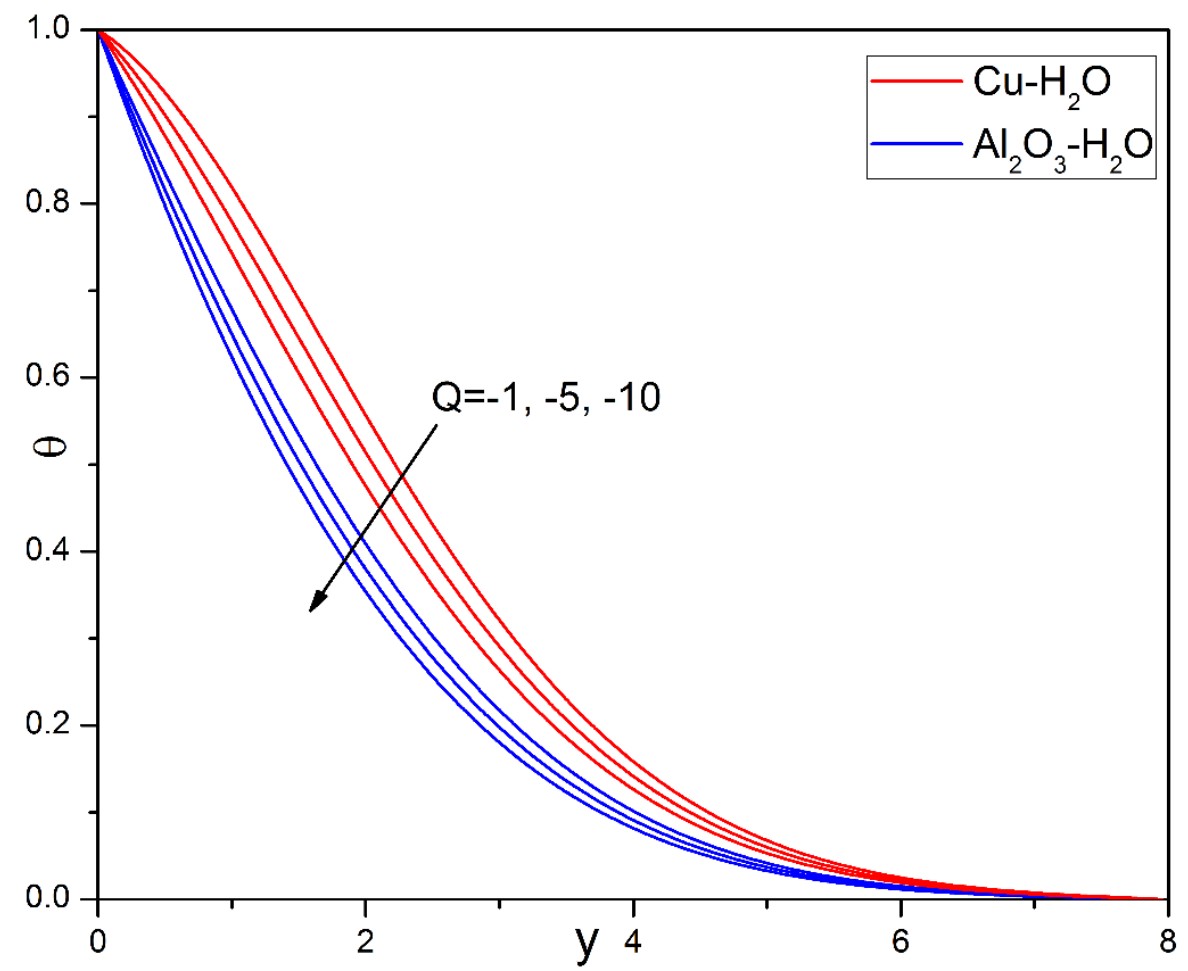

Figure 5: The influence of heat sink on temperature profiles.

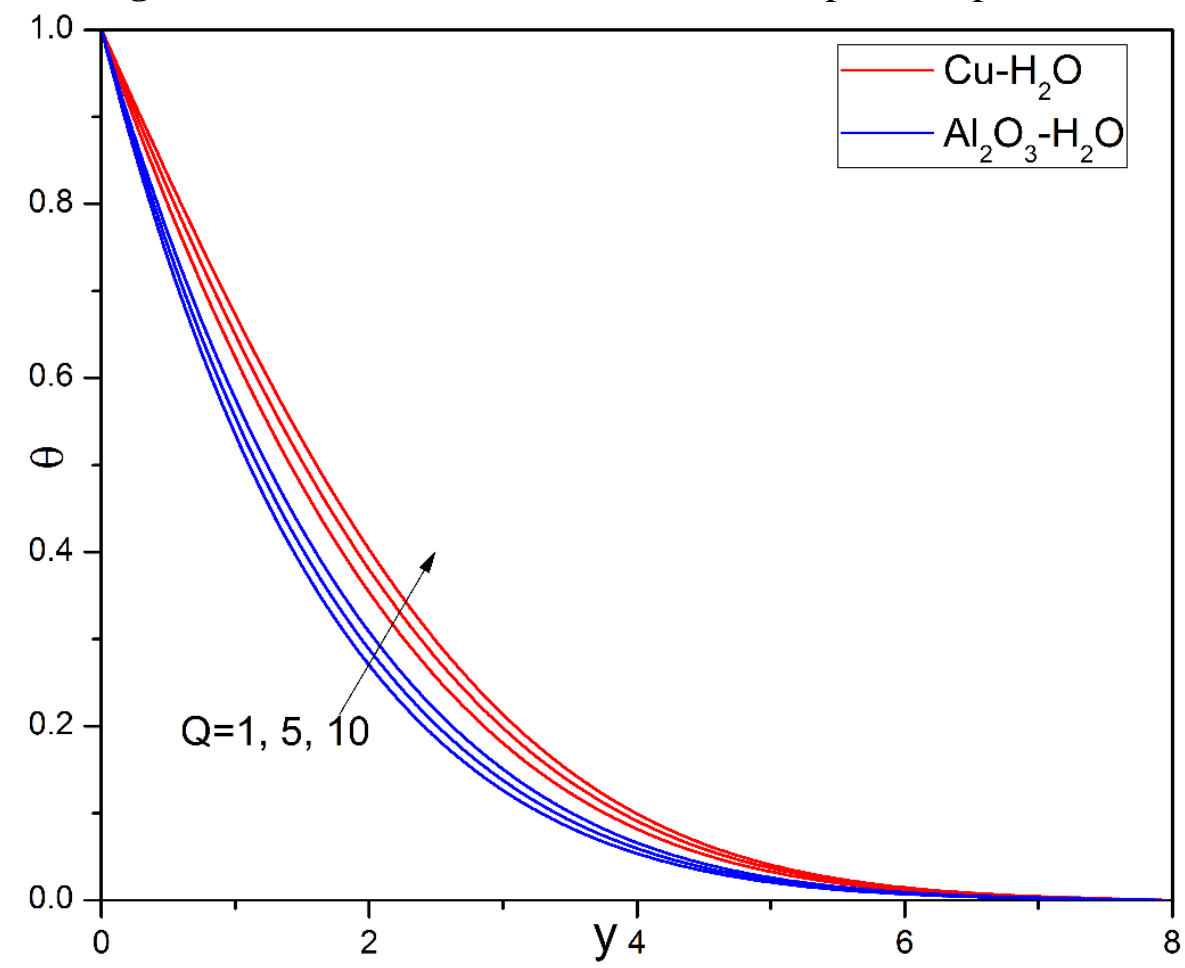

Figure 6: The influence of heat source on temperature profiles. 


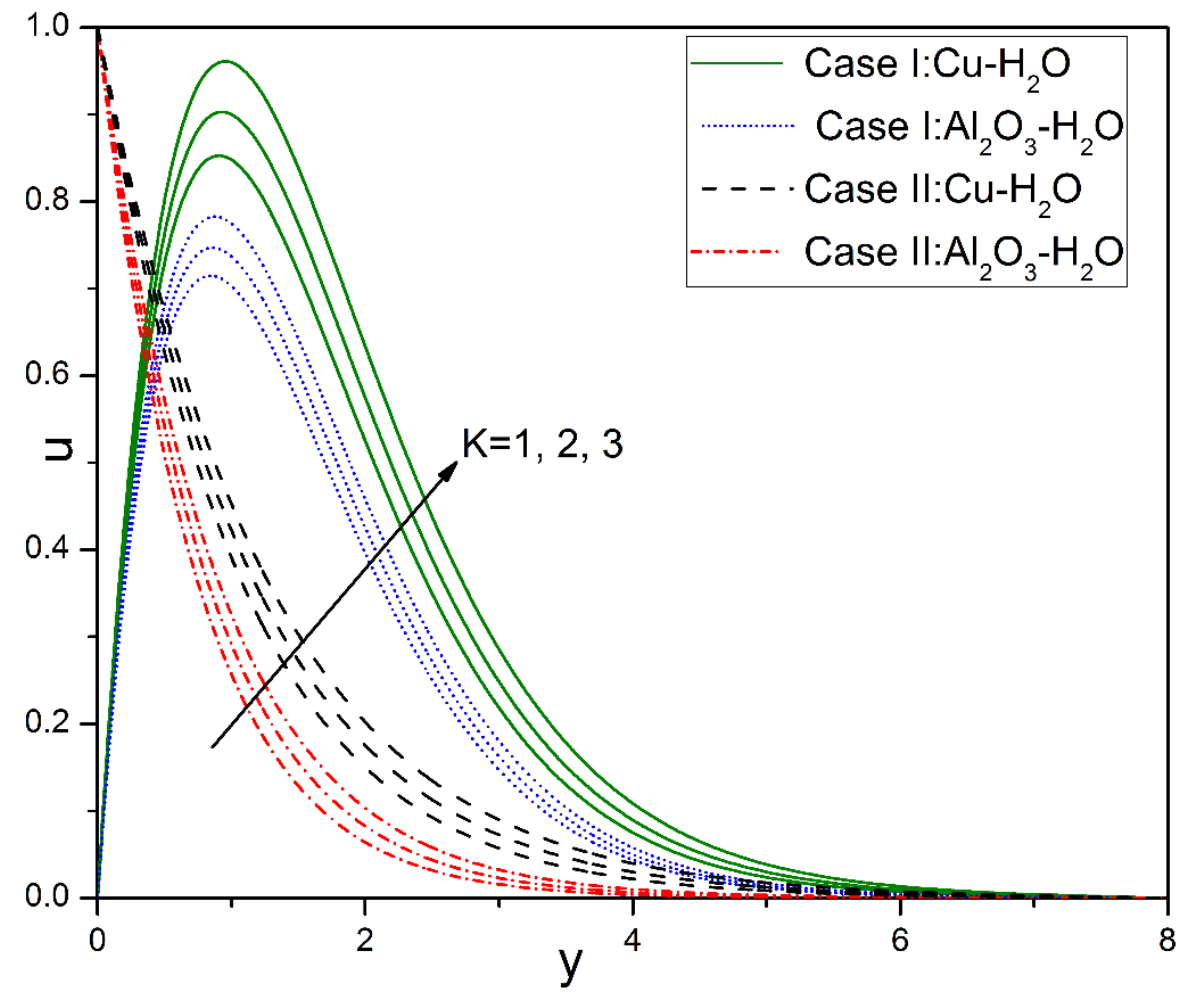

Figure 7: The influence of permeability parameter on velocity profiles.

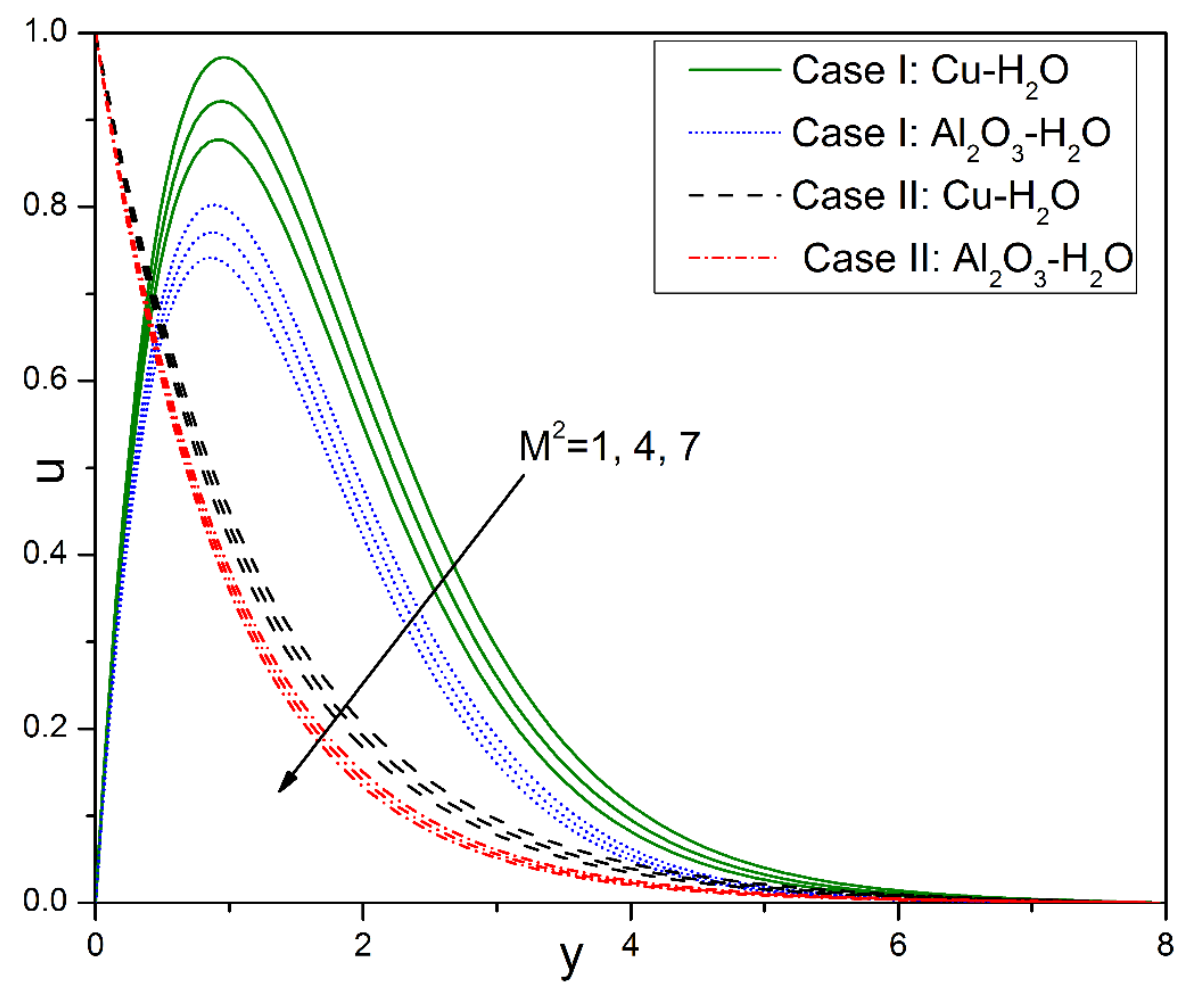

Figure 8: The influence of Hartmann magnetic number on velocity profiles. 


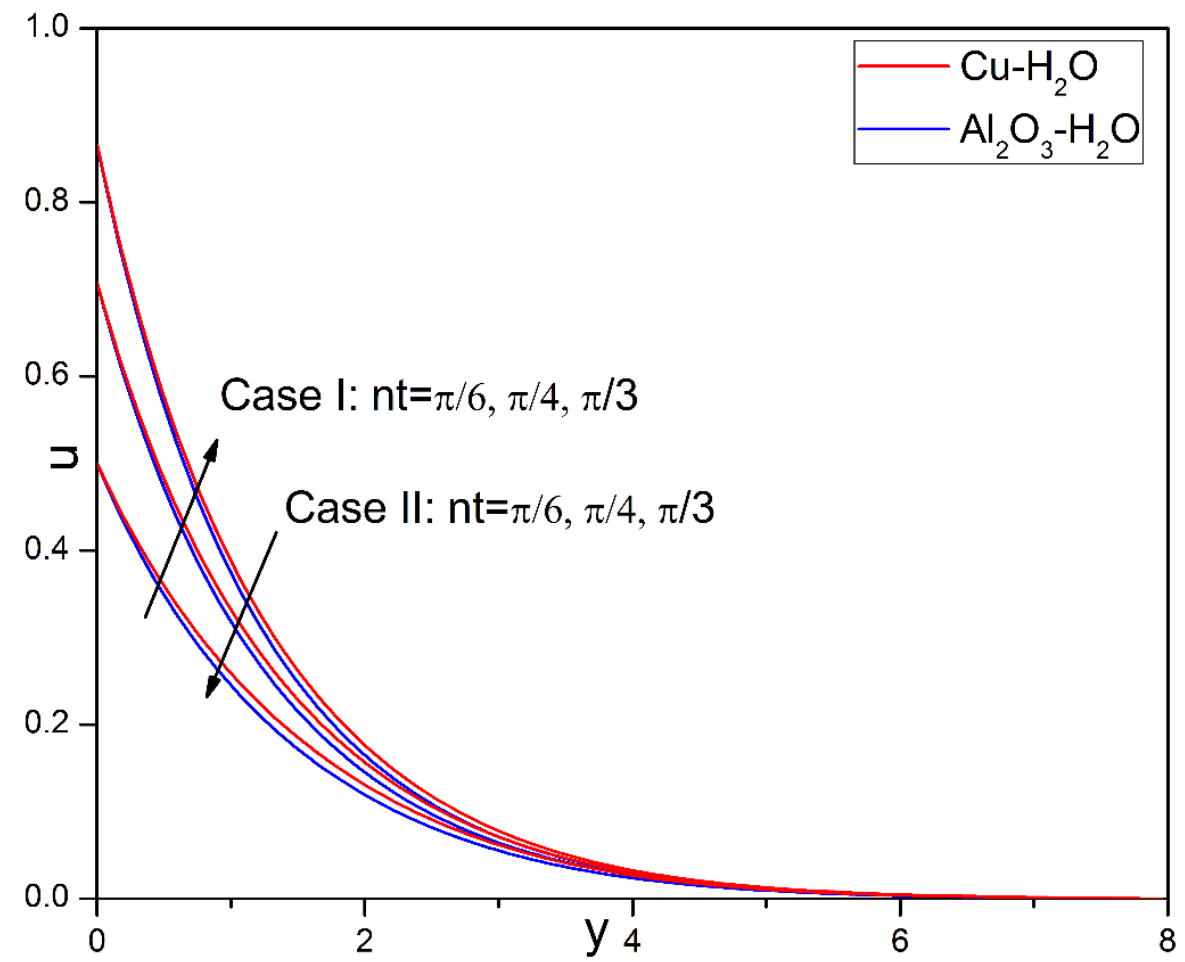

Figure 9: The influence of $n t$ on velocity profiles.

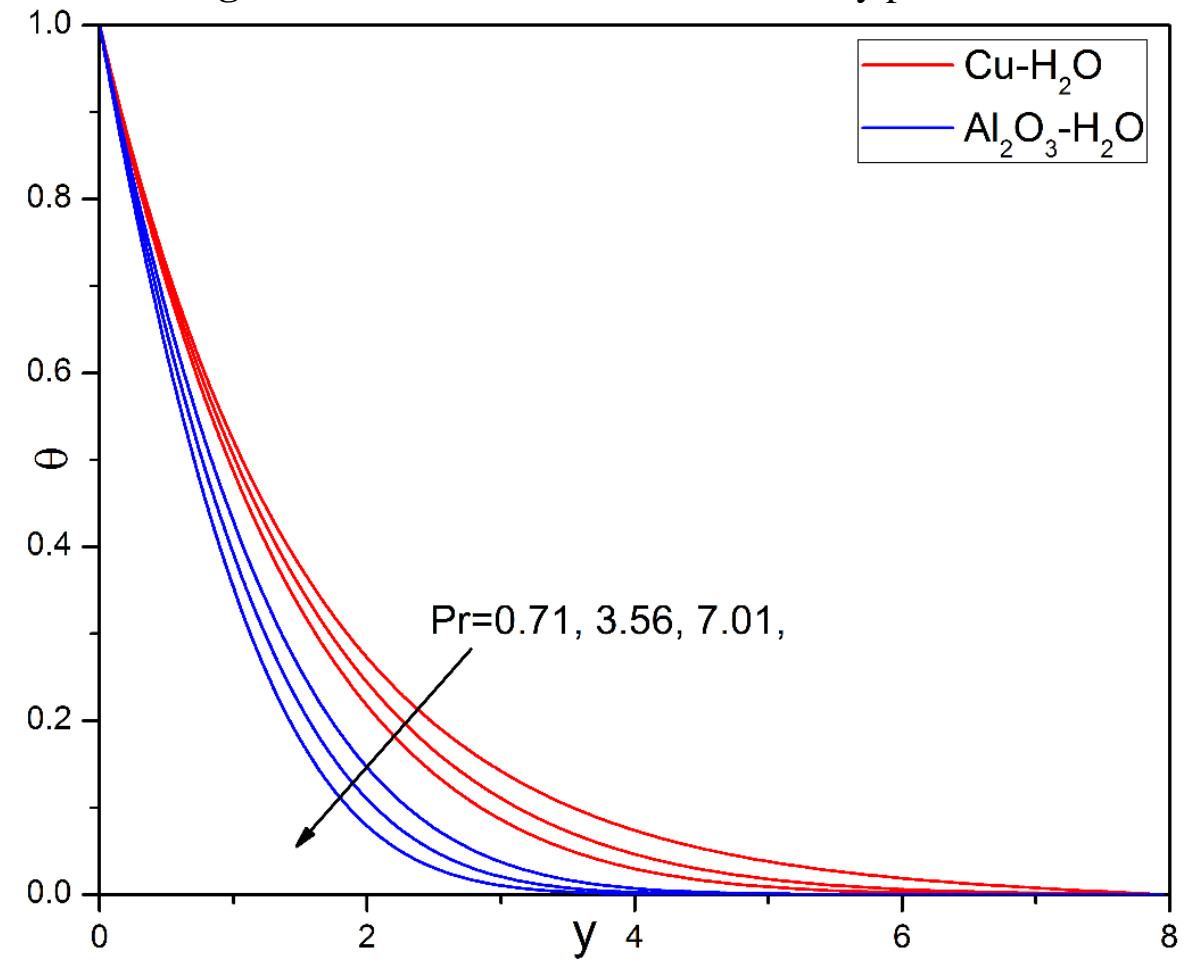

Figure 10: The influence of Prandtl number on temperature profiles. 


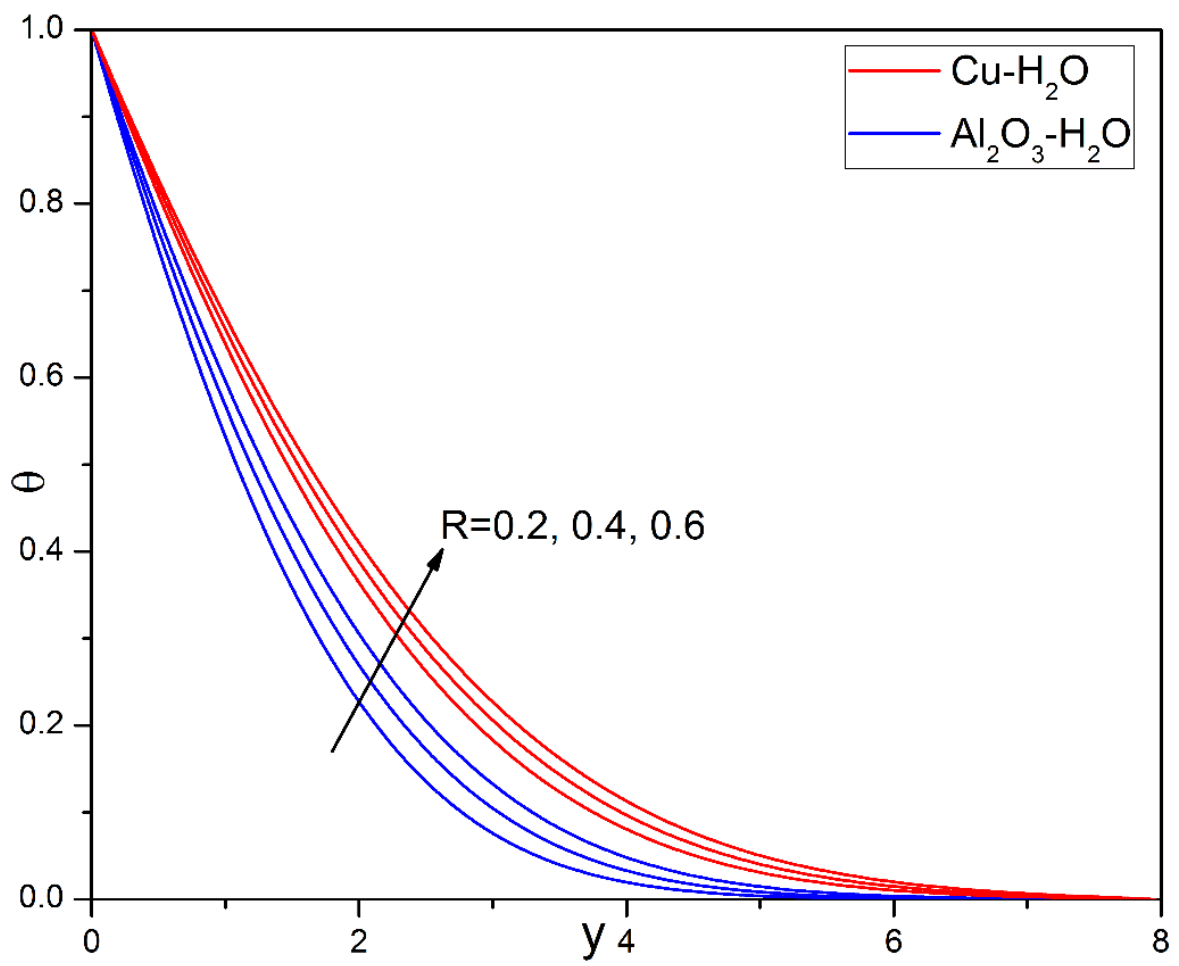

Figure 11: The influence of radiation-conduction parameter on temperature profiles.

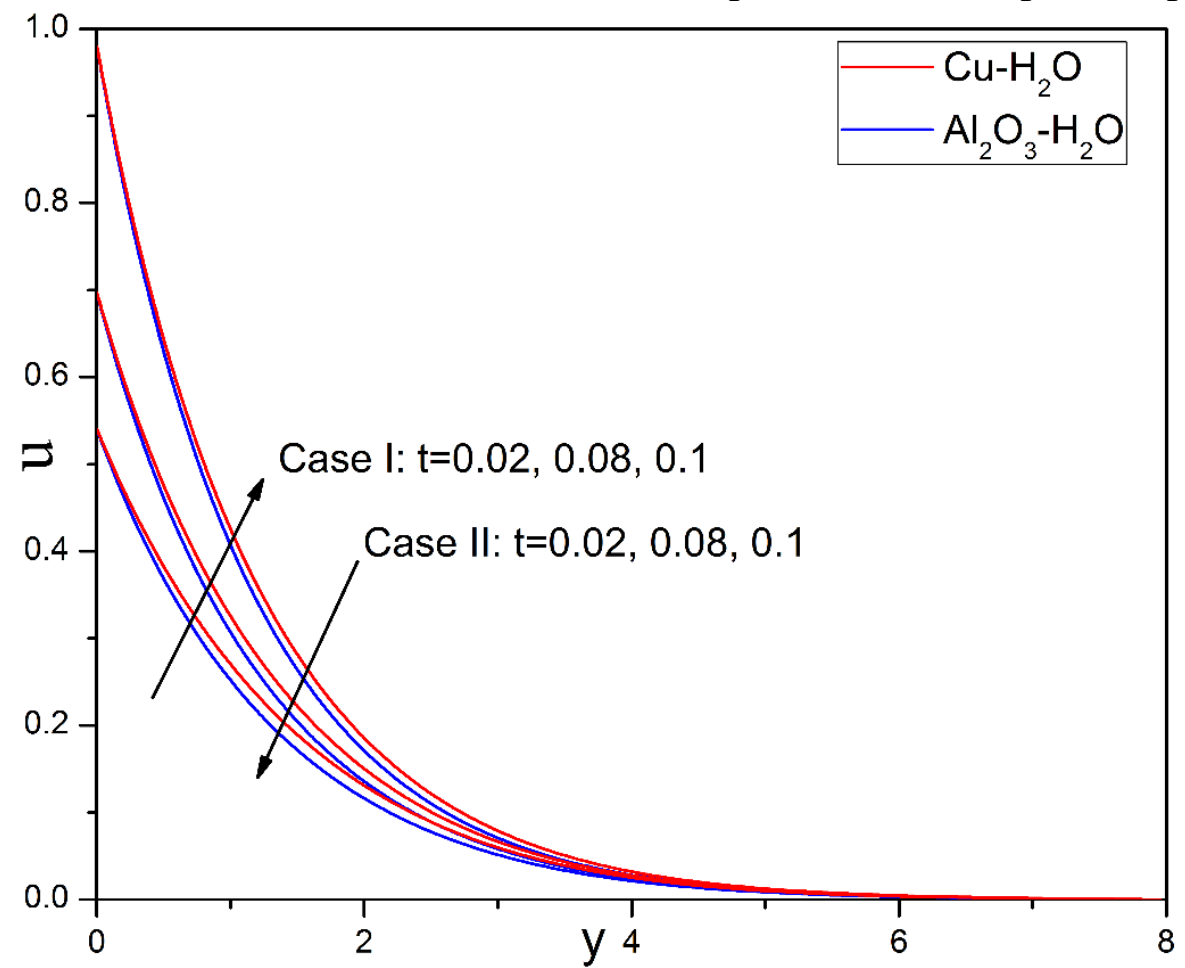

Figure 12: The influence of time parameter on velocity profiles. 


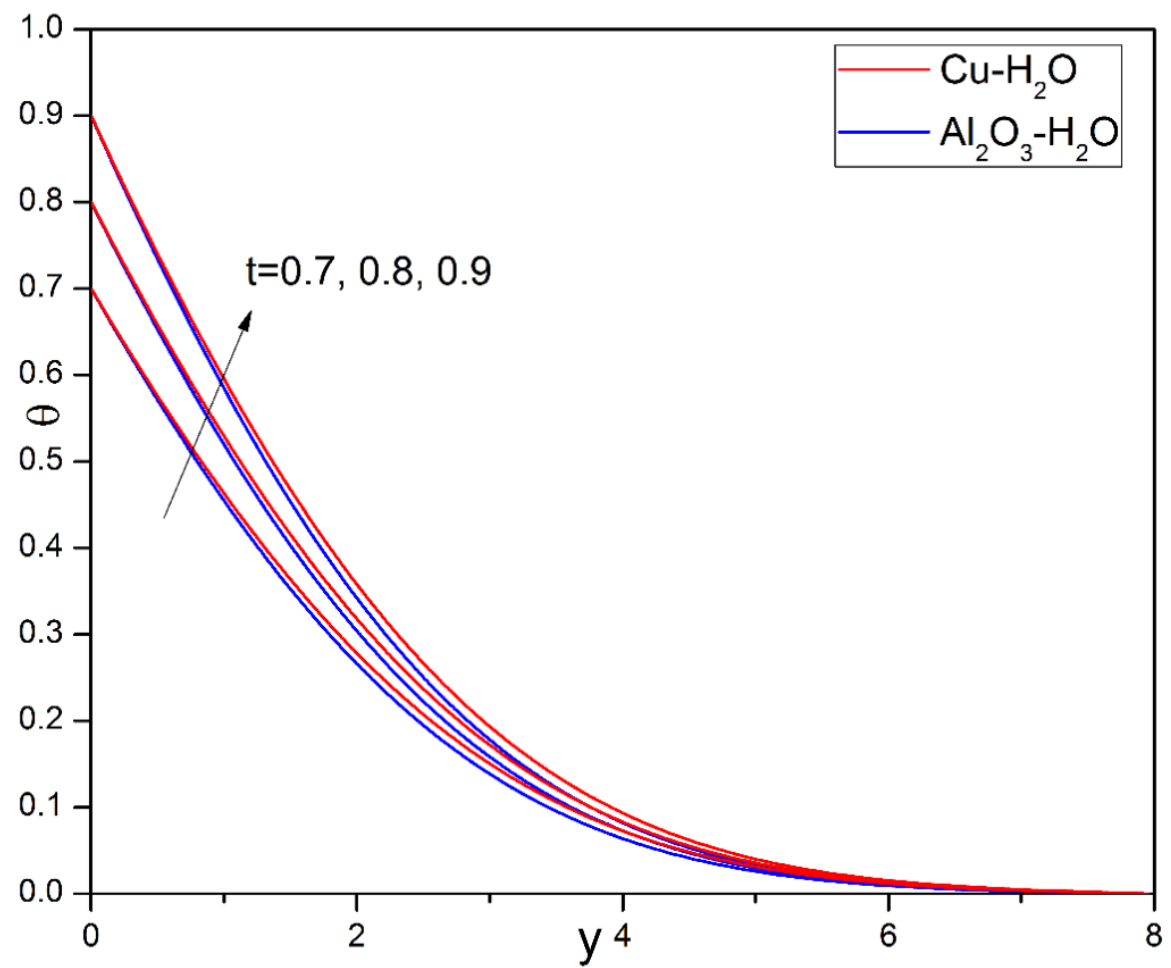

Figure 13: The influence of time parameter on temperature profiles.

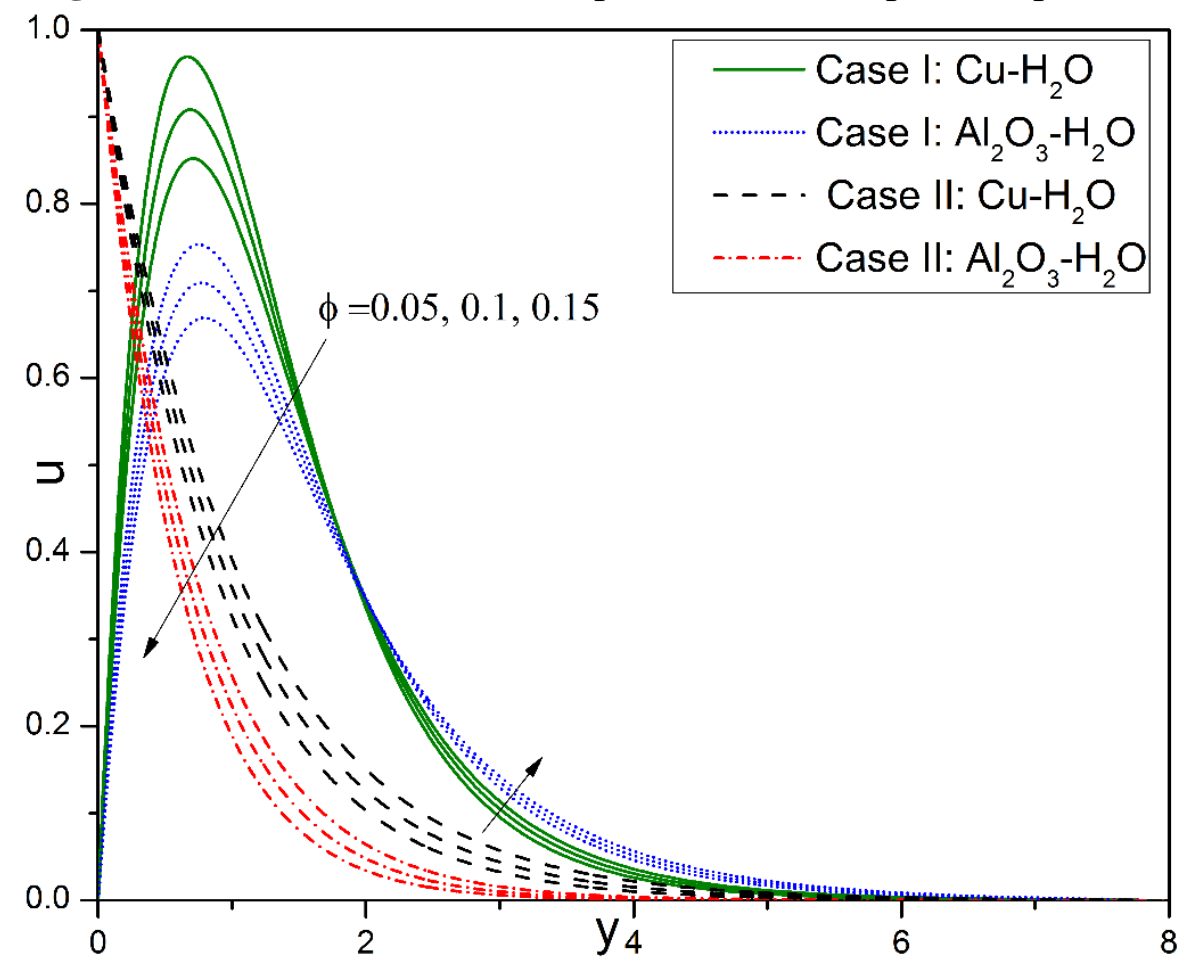

Figure 14: The influence of volume fraction on velocity profiles. 


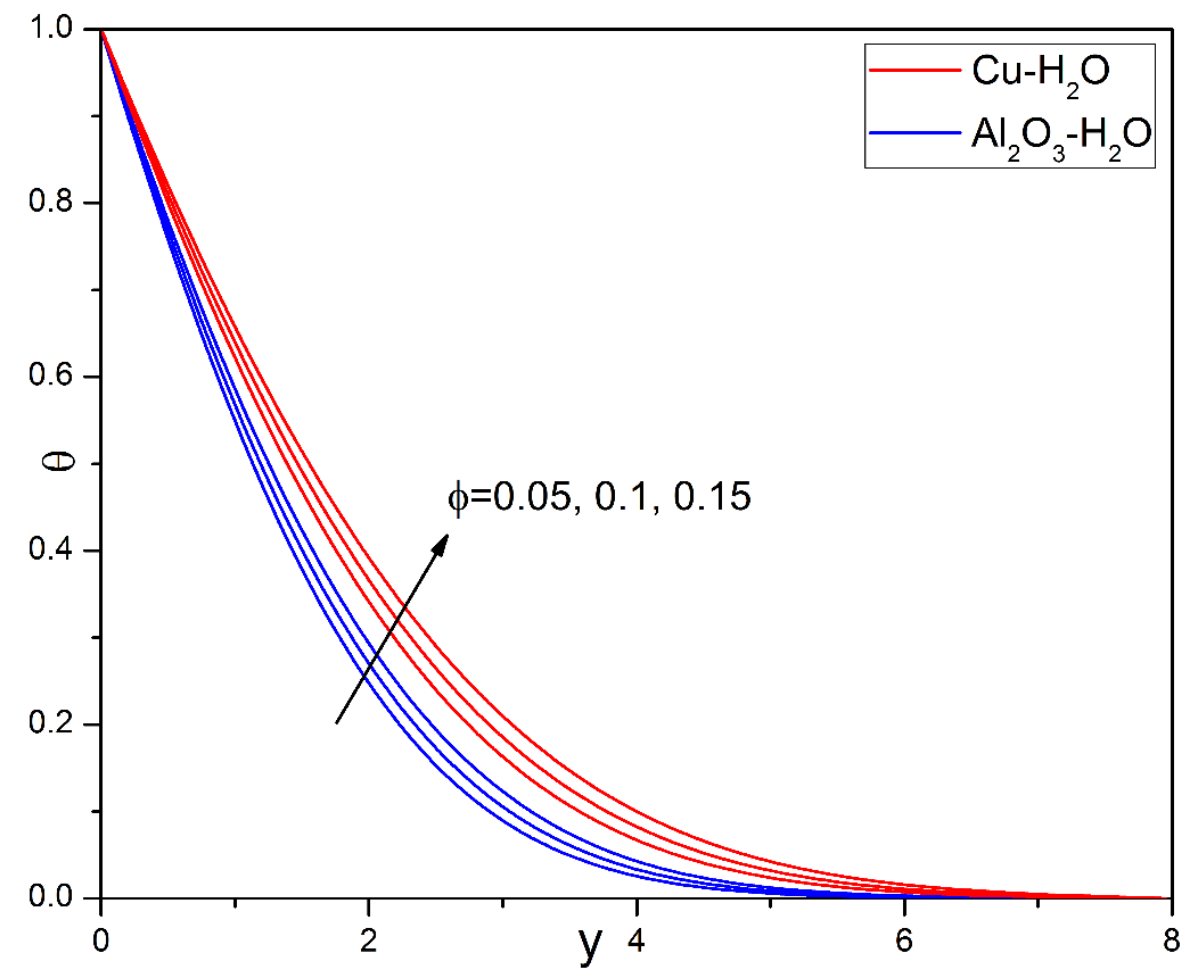

Figure 15: The influence of volume fraction on temperature profiles.

The temperature profiles for exceed consistently those of $\mathrm{Cu}-\mathrm{H}_{2} \mathrm{O}$ when compared with the $\mathrm{Al}_{2} \mathrm{O}_{3}-\mathrm{H}_{2} \mathrm{O}$ nanofluid profiles, this is again due to the high thermal conductivity of $\mathrm{Cu}$ than that of $\mathrm{Al}_{2} \mathrm{O}_{3}$ which in turn leads to the enhancement of the thermal boundary layer for $\mathrm{Cu}-\mathrm{H}_{2} \mathrm{O}$ relative to the other nanofluid. The asymptotically smooth nature of profiles in the free stream i.e. large $y$ values confirm that an adequately large infinity boundary condition has been prescribed in the finite element code. It is clearly observed from Figure 2 that velocity is decreased with an increase of angle of inclination. This is generally due to the reduction in the thermal buoyancy force which is scaled according to $\cos (\alpha)$. As the plate inclination rises, this buoyancy force component, $-A_{2} G r \theta \cos (\alpha)$ in equation (9), is progressively reduced from unity (for $\alpha=0$ i.e. vertical plate, for which $\cos (\alpha)=1$ ) to zero (for $\alpha=\pi / 2$ i.e. horizontal plate for which $\cos (\alpha) \rightarrow 0$ i.e. eliminating thermal buoyancy and gravity effect so that the flow becomes purely forced convection-radiation in this limit). The reduction in buoyancy force between these two limits manifests in a decrease in thermal diffusion which opposes momentum development. Here we have considered intermediate values of inclination angle i.e. $\pi / 6, \pi / 4, \pi / 3$ and clearly the fluid attains a higher velocity for $\alpha=\pi / 6$ (stronger thermal buoyancy) and a minimum velocity for $\alpha=\pi / 3$ (weaker thermal buoyancy). Consequently, momentum boundary layer thickness is increased as a result of decrease in the velocity of nanofluids. Momentum boundary layer thickness is 
lowest for $\alpha=\pi / 6$ (stronger thermal buoyancy) and highest for $\alpha=\pi / 3$ (weak buoyancy). The trends in the results are also consistent with other studies including Rana and Beg [34].

Figures 3 and 4 illustrate the velocity evolution with variation in thermal Grashof number $G r$. This parameter as with plate inclination also arises in the thermal buoyancy force, $-A_{2} G r \theta \cos (\alpha)$ in the dimensionless momentum equation (9). Grashof number quantifies the relative magnitude of the buoyancy force and the opposing frictional (viscous hydrodynamic) forces acting on the magnetic nanofluid. Physically the positive, negative and zero values of the Grashof number represents the cooling, heating of the boundary surface and absence of free convection currents, respectively. The velocity profiles in case of sinusoidal and co-sinusoidal oscillations are increasing for $G r>0$ with the increase of thermal Grashof number and this is due to the dominance of buoyancy forces over the viscous forces, which in turn energizes and accelerates the flow, manifesting in an increase in velocities. The opposite trend is observed when $G r<0$. It is evident from the graphs that the velocity profiles in case II starts with the velocity of the plate and gradually decreases to zero at a sufficiently far from the boundary layer and in case I velocity increases with distance from the surface, reaches to maximum value in the vicinity of the plate, and decrease monotonically to zero at the free stream. It is also pertinent to note that for $G r>0$ (plate cooling) a velocity overshoot is observed near the plate surface which is absent for $G r<0$ (plate heating). The general effect of Grashof number however is sustained for the two different cases. Thermal buoyancy clearly exerts a very significant influence in the regime.

Figures 5 and 6 depict the response in nanofluid temperature distributions across the boundary layer to various values of heat source $(Q>0)$ and heat $\operatorname{sink}(Q<0)$. It is clear from the graphs that all the nanofluid temperature profiles decrease for $Q<0$, this is due to the fact that when heat is removed from the regime, the nanofluid is de-energized. Similar observations have been made by Chamkha and Ali [38] and Chamkha et al. [40]. Internal heat absorption (sink) therefore maybe exploited to successfully cool the regime. The opposite trend is observed for heat source $(Q>0)$ indicating that with internal heat generation, temperatures are strongly elevated. Therefore thermal boundary layer thickness is enhanced with increasing heat source $(Q>0)$ whereas it is reduced with greater heat sink ( $Q<0)$. In magnetic nanofluid processing operations, therefore a significant change in temperatures can be achieved with a simple heat sink or source mechanism as elaborated further in [55]. Indeed to this effect, trailing heat sinks have been shown to be very effective in reducing temperatures in thin sheet fabrication systems [56]. Furthermore a travelling (rather than static) heat source may also be very beneficial to achieving the opposite effect in thermal materials processing as elucidated by Doumanidis and Fourligkas [57] and this will be explored in future simulations.

Figure 7 illustrates the influence of the permeability parameter, $K$ on velocity evolution. Based on the viscous-dominated Darcy model [58], the fiber resistance to nanofluid transport in the regime external to the plate is simulated via the linear resistance term, $-A_{3}\left(\frac{1}{K}\right) u$ in the momentum equation (9). The body force is inversely proportional to permeability parameter. As $K$ increases, corresponding to a decrease in presence of solid fibers in the porous medium, the Darcian impedance is progressively reduced. This results in acceleration in the flow and a decrease in momentum boundary layer thickness. Very large values of $K$ are considered as the analysis considers sparsely packed permeable materials which are utilized 
in real materials processing systems [59]. The graphs further illustrate the effect of permeability parameter on the velocity distributions for two water based nanofluids in the case of sinusoidal and co-sinusoidal oscillations. The $\mathrm{Cu}-\mathrm{H}_{2} \mathrm{O}$ nanofluid consistently achieves greater velocities than $\mathrm{Al}_{2} \mathrm{O}_{3}-\mathrm{H}_{2} \mathrm{O}$ nanofluid for both oscillatory cases. However for Case I evidently, as in other plots, a much greater velocity is computed further into the boundary layer away from the plate, whereas for Case II the velocity is greater at and in the vicinity of the plate.

Figure 8 presents the influence of the Hartmann magnetic number $(M)$ on velocity profiles in the regime. The presence of a magnetic field transverse to the plate surface generates a Lorentzian magnetic drag force, $-A_{3}\left(M^{2}\right) u$ which acts in the reverse direction to the flow.

This magnetic drag is proportional to the magnetic parameter $M^{2}=\frac{\sigma_{f} B_{o}^{2} v_{f}}{\rho_{f} u_{o}^{2}}$, an increase in $M^{2}$ therefore enhances the magnetohydrodynamic drag which decreases the velocity and decelerates the flow. This leads to a growth in momentum boundary layer thickness. Again it is apparent that $\mathrm{Cu}-\mathrm{H}_{2} \mathrm{O}$ nanofluid achieves better acceleration than $\mathrm{Al}_{2} \mathrm{O}_{3}-\mathrm{H}_{2} \mathrm{O}$ nanofluid although greater differences are computed for Case I (sinusoidal oscillations) as compared to Case II (co-sinusoidal oscillations). As found in other plots, higher velocity is observed further into the boundary layer away at some distance from the plate, whereas for Case II a higher velocity is computed much closer to the plate and at the plate.

Figure 9 illustrates the distributions of velocity with different modified time parameter values $(n t)$ which incorporates the oscillation frequency $n$. In all cases, maximum velocity is observed at the plate surface itself. Profiles consistently descend monotonically from the plate to the free stream, irrespective of $n t$ value or nanofluid. However for Case I (sinusoidal oscillations), with increasing $n t$ values i.e. greater progression in time, there is a significant acceleration induced in the flow. Conversely for Case II (co-sinusoidal oscillations) the opposite behavior is computed and there is a deceleration with greater $n t$ values i.e. the flow is damped considerably. In both Cases I and II, $\mathrm{Cu}-\mathrm{H}_{2} \mathrm{O}$ nanofluid attains slightly greater velocity magnitudes than $\mathrm{Al}_{2} \mathrm{O}_{3}-\mathrm{H}_{2} \mathrm{O}$ nanofluid and this trend is most prominent further from the plate. It is furthermore noteworthy that despite a strong deceleration of the flow in Case II, backflow i.e. flow reversal is not induced as testified to by the consistently positive values of the velocity $(u)$.

Figure 10 presents the variations in the temperature profiles for different values of Prandtl number(Pr). An increase in Prandtl number generates a substantial decrease in temperatures. This leads to a concomitant reduction in thermal boundary layer thickness. Prandtl number defines the relative contribution of momentum diffusion to thermal diffusion in the boundary layer regime. For $\operatorname{Pr}<1$ momentum diffusion is exceeded by thermal diffusion and the opposite applies for $\operatorname{Pr}>1$. Only when $\operatorname{Pr}=1$ do both momentum and energy diffuse at the same rate and the hydrodynamic and thermal boundary layer thicknesses become equivalent in magnitude. Prandtl number is also inversely proportional to thermal conductivity. Lower Prandtl number nanofluids (magnetic liquid metal nanofluids are an extreme case for ( $\operatorname{Pr}<<1)$ possess greater thermal conductivities which result in enhanced thermal diffusion and greater temperatures. Conversely higher Prandtl number liquids (polymers are an extreme case where $(\operatorname{Pr}>>1)$ have much lower thermal conductivities and this results in lower temperature magnitudes. With a decrease in $\operatorname{Pr}$, heat is able to diffuse away from the heated 
surface more rapidly than at higher values of $\operatorname{Pr}$. Hence the rate of heat transfer is also reduced. Furthermore an increase in Pr causes a marked decrease in thickness of the thermal boundary layer. Very smooth convergence of solutions is again observed in the free stream, confirming the imposition of a sufficiently large infinity boundary condition in the finite element computations. Again it is evident also that $\mathrm{Cu}-\mathrm{H}_{2} \mathrm{O}$ nanofluid achieves notably greater temperatures than $\mathrm{Al}_{2} \mathrm{O}_{3}-\mathrm{H}_{2} \mathrm{O}$ nanofluid, and this is due to the contribution of a greater thermal conductivity of copper metallic nano-particles compared with aluminum.

Figure 11 depict the temperature distributions for various conduction-radiation parameter values $(\mathrm{R})$, again for two selected water-based nanofluids $\left(\mathrm{Cu}-\mathrm{H}_{2} \mathrm{O}\right.$ and $\mathrm{Al}_{2} \mathrm{O}_{3}-\mathrm{H}_{2} \mathrm{O}$ ). It is evident from the figures that with an increase in $R$ values, temperatures are enhanced strongly for both nanofluids. However significantly larger temperatures are computed for $\mathrm{Cu}-\mathrm{H}_{2} \mathrm{O}$ nanofluid compared with $\mathrm{Al}_{2} \mathrm{O}_{3}-\mathrm{H}_{2} \mathrm{O}$ nanofluid. The parameter, $\mathrm{R}$, arises in the augmented thermal diffusion term, $A_{5} \frac{\partial^{2} \theta}{\partial y^{2}}$ in the energy conservation equation (10), where $A_{5}=A_{4}\left(K_{n f}+R\right)$ and $R=\frac{16 \sigma^{*} T_{\infty}^{* 3}}{3 k k^{*}}$. Effectively $R$ represents the relative role of thermal radiation and thermal conduction heat transfer modes. For $R<1$ thermal conduction dominates and for $R>1$ thermal radiation dominates. It is more physically viable to consider the former. For $R=1$ (not considered both thermal radiation and conduction contribute equally). As $R$ is increased from 0.2 , through 0.4 and then 0.6 , the contribution of radiative transfer relative to conductive heat transfer is first doubled and then tripled. This imparts significant heat to the flow regime, energizes the boundary layer and elevates temperatures for all values of $y$. The presence of thermal radiation, as anticipated, is therefore very substantial on the variation of temperature. Thermal boundary layer thickness is therefore strongly increased with increasing $R$ values. These trends are consistent with many other investigations reported on radiative-convective nanofluid flows including Das and Jana [17] and Gireesha et al. [20].

Figures 12 to 13 distributions of temperature with different time parameter values $(t)$. In Figure 12 we consider both oscillatory plate cases. Although in both Case I and II, it is apparent that the flow is accelerated with time progression, the effect is more profound for Case I than Case II. Elapse in time allows a development in momentum in the boundary layer regime and this allows the escalation in velocities, which are computed at all distances from the plate. There is no onset of backflow. Velocities clearly decay gradually from the plate to the free stream with increasing transverse coordinate, $y . \mathrm{Cu}-\mathrm{H}_{2} \mathrm{O}$ nanofluid demonstrates distinctly higher velocities than $\mathrm{Al}_{2} \mathrm{O}_{3}-\mathrm{H}_{2} \mathrm{O}$ nanofluid at all-time instants and locations and for both Cases. In Figure 13 we observe that generally again progression in time leads to a substantial acceleration in the flow and once again $\mathrm{Cu}-\mathrm{H}_{2} \mathrm{O}$ nanofluid achieves better acceleration than $\mathrm{Al}_{2} \mathrm{O}_{3}-\mathrm{H}_{2} \mathrm{O}$ nanofluid. Momentum boundary layer thickness is therefore decreased with time and is greater for $\mathrm{Al}_{2} \mathrm{O}_{3}-\mathrm{H}_{2} \mathrm{O}$ nanofluid compared with $\mathrm{Cu}-\mathrm{H}_{2} \mathrm{O}$ nanofluid.

Figures 14 and 15 illustrate the impact of nano-particle volume fraction $(\phi)$ on respectively the velocity field (for both Case I and Case II) and the temperature function $(\theta)$. As volume 
fraction increases, the thermal conductivity of nanofluid is elevated. Therefore thermal diffusion is assisted in the regime. Velocity magnitudes are strongly decreased with increasing $(\phi)$ values as seen in Figure 14 for Case I. Although $\mathrm{Cu}-\mathrm{H}_{2} \mathrm{O}$ nanofluid velocity is greater initially than $\mathrm{Al}_{2} \mathrm{O}_{3}-\mathrm{H}_{2} \mathrm{O}$ nanofluid velocity, with progressive distance along the plate i.e. greater $y$ values, this trend is reversed and $\mathrm{Al}_{2} \mathrm{O}_{3}-\mathrm{H}_{2} \mathrm{O}$ nanofluid velocity weakly exceeds $\mathrm{Cu}-\mathrm{H}_{2} \mathrm{O}$ velocity far from the leading edge i.e. closer to the freestream. Here $\mathrm{Cu}-\mathrm{H}_{2} \mathrm{O}$ nanofluid again achieves consistently greater velocity magnitudes for both Case I (sinusoidal oscillation) and Case II (co-sinusoidal oscillation) as compared with $\mathrm{Al}_{2} \mathrm{O}_{3}-\mathrm{H}_{2} \mathrm{O}$ nanofluid. Figure 15 indicates that a very considerable increase in temperatures is attained with increasing nanoparticle volume fraction $(\phi)$. Thermal conductivity of waterbased nanofluids increases as the nanoparticle size increases since the low viscosity of the base fluid promotes the clustering of nanoparticles. This particle clustering forms interconnecting channels for thermal energy to propagate. As a result as nanoparticle volume fraction increases the thermal conductivity of water-based nanofluid is enhanced and greater thermal energy imparted to the nanofluid boundary layer. This elevates temperatures and also increases thermal boundary layer thickness.

\section{Conclusions}

Motivated by high temperature nano-materials processing operations, a mathematical and computational study has been conducted to investigate radiative magnetohydrodynamic convective Newtonian nanofluid transport phenomena from an oscillating inclined porous plate with variable temperature. Internal heat generation (source)/heat absorption (sink) and thermal buoyancy effects have been incorporated. The Tiwari-Das nanofluid model which uses a nanoparticle volume fraction has been adopted. Four different metallic spherical shaped nano-particles have been explored i.e. silver oxide, copper, aluminium oxide and titanium oxide, suspended in water base fluid. The Brinkman and Maxwell-Garnetts models have been employed for simulating dynamic viscosity and effective thermal conductivity of the nanofluids respectively. The Rosseland diffusion approximation which is valid for optically thick materials has been used to simulate thermal radiative flux effects. A linear Darcy model has been utilized to mimic porous medium drag force effects. Finite element numerical solutions have been obtained for the transformed coupled, multi-physical boundary value problem based on the weak variational formulation and weighted residual scheme with appropriate initial and boundary conditions. A grid independence study has been included and validation of special cases of the general model against existing solutions in the published literature also conducted. The current model has extended existing simulations by considering plate inclination, heat generation and also combined magnetohydrodynamic and radiative effects in unsteady flow in porous materials. The present computations have shown that:

- With increasing Prandtl number and time, the skin friction i.e. shear stress at the plate surface is increased for all nanofluids.

- With increasing Prandtl number and time, Nusselt number is generally increased for any nanofluid.

- Skin friction is minimized for $\mathrm{Ag}-\mathrm{H}_{2} \mathrm{O}$ nanofluid, and maximum for $\mathrm{Al}_{2} \mathrm{O}_{3}-\mathrm{H}_{2} \mathrm{O}$ nanofluid. 
- Nusselt number (wall heat transfer rate) is a maximum for $\mathrm{Cu}-\mathrm{H}_{2} \mathrm{O}$ nanofluid, closely followed by $\mathrm{Ag}-\mathrm{H}_{2} \mathrm{O}$, then $\mathrm{Al}_{2} \mathrm{O}_{3}-\mathrm{H}_{2} \mathrm{O}$ and finally lowest for $\mathrm{TiO}_{2}-\mathrm{H}_{2} \mathrm{O}$ nanofluids.

- An increase in nanoparticle volume fraction, heat source (generation) and radiationconduction parameter enhances temperatures and thermal boundary layer thicknesses whereas an increase in Prandtl number and heat sink (absorption) induces the opposite effect.

- The flow is decelerated with an increase of angle of inclination owing to a reduction in thermal buoyancy force.

- The velocity profiles for both sinusoidal and co-sinusoidal oscillations are enhanced for positive Grashof number (plate cooling) whereas they are decreased for negative Grashof number (plate heating).

- An increase in nanoparticle volume fraction and Hartmann magnetic parameter both decrease velocity and increase momentum boundary layer thickness whereas an increase in permeability parameter induces the opposite effect and accelerates the flow with a reduction in momentum boundary layer thickness.

- Generally $\mathrm{Cu}-\mathrm{H}_{2} \mathrm{O}$ achieves greater velocity and temperature magnitudes than $\mathrm{Al}_{2} \mathrm{O}_{3}-\mathrm{H}_{2} \mathrm{O}$ nanofluid at any location and any time instant.

- For both sinusoidal and co-sinusoidal oscillations of the plate (i.e. Cases I, II), the flow is accelerated with increasing time.

- With greater modified time parameter values which incorporates the oscillation frequency, the nanofluid is accelerated for Case I (sinusoidal oscillations), whereas it is decelerated for Case II (co-sinusoidal oscillations).

- The present simulation has been confined to Newtonian viscous flow. Future investigations will consider non-Newtonian nanofluid models e.g. viscoelastic [60] and will be communicated soon.

\section{Acknowledgement}

The authors are thankful to the reviewers for their insightful comments on our research paper which have improved aspects of the work.

\section{References}

1. Choi S. Enhancing thermal conductivity of fluids with nanoparticles, in Developments and Applications of non-Newtonian flows. Siginer DA, Wang HP, editors, ASME 1995; 66: 99-105.

2. Choi SUS, Zhang ZG, Yu W, Lockwood FE and Grulke EA. Anomalously thermal conductivity enhancement in nanotube suspensions. Appl. Phys. Lett 2001; 79: 2252.

3. Haddad Z, Oztop HF, Abu Nada E and Mataoui A. A review on natural convective heat transfer of nanofluids, Renewable and Sustainable Energy Reviews 2012; 16: 5363.

4. Buongiorno J. Convective transport in nanofluids. ASME J Heat Transfer 2006; 128: 240-250. 
5. Tiwari RK and Das MK. Heat transfer augmentation in a two-sided lid-driven differentially heated square cavity utilizing nanofluids. Int. J Heat Mass Transfer 2007; 50: 2002-2018.

6. Jin ZQ and Liu JP. Rapid thermal processing of magnetic materials. J. Phys. D: Appl. Phys. 2006; 39:R227.

7. Schneider-Muntau HJ and Wada H. Materials Processing in Magnetic Fields, Proc. Int. Workshop on Materials Analysis and Processing in Magnetic Fields. Tallahassee, Florida, 17 - 19 March (2004).

8. Fautrelle Y, Ernst R and Moreau R. Magnetohydrodynamics applied to materials processing. Int. J. Materials Research 2009; 100(10):1389-1398.

9. Li H, Fautrelle Y, Hou L and et al. Effect of a weak transverse magnetic field on the morphology and orientation of directionally solidified Al-Ni alloys. J. Crystal Growth 2015; 436: 68-75.

10. Bég O. Anwar, Bakier AY, Prasad VR and Ghosh SK. Non similar, laminar, steady, electrically-conducting forced convection liquid metal boundary layer flow with induced magnetic field effects. Int. J. Thermal Sciences 2009; 48(8): 1596-1606.

11. Zueco J, Bég O. Anwar, Takhar HS. Network numerical analysis of magneto-micropolar convection through a vertical circular non-Darcian porous medium conduit. Computational Materials Science 2009; 46(4): 1028-1037.

12. Tsung TT and et al. A novel nanofluid manufacturing process using a cylindrical flow cooling method in an induction system. Int. J. Advanced Manufacturing Technology 2006; 29: 99-104.

13. Zhao B and Nan Z, Preparation of stable magnetic nanofluids containing Fe3O4@PPy nanoparticles by a novel one-pot route. Nanoscale Research Letters 2011; 6: 230-235.

14. Mahanthesh B, Gireesha BJ and Gorla RSR. Heat and mass transfer effects on the mixed convection flow of chemically reacting nanofluid past a moving/stationary vertical plate. Alexandria Engineering J. 2016; 55: 569.

15. Akbar NS, Tripathi D and Bég O. Anwar. Modelling nanoparticle geometry effects on peristaltic pumping of medical magnetohydrodynamic nanofluids with heat transfer. $J$. Mechanics in Medicine and Biology 2016; http://dx.doi.org/10.1142/S0219519416 500883

16. Thiagarajan M and Selvaraj M. Nonlinear MHD stagnation point flow and heat transfer of diamond, platinum - mineral oil based nanofluid over a flat plate with viscous dissipation. J. Nanofluids 2016; 5: 231.

17. Das $\mathrm{S}$ and Jana RN. Natural convective magneto-nanofluid flow and radiative heat transfer past a moving plate. Alexandria Engineering $J$ 2014; 54: 55.

18. Siva Reddy $S$ and Thirupathi T. Heat and mass transfer effects on natural convection flow in the presence of volume fraction for copper-water nanofluid. J. Nanofluids 2016; 5: 220.

19. Ferdows M, Khan MS, Bég O. Anwar et al. Numerical study of transient magnetohydrodynamic radiative free convection nanofluid flow from a stretching permeable surface. Proc. IMECHE-Part E: J. Process Mechanical Engineering 2014; 228(3): 181-196.

20. Gireesha BJ, Gorla RSR and Mahanthesh B. Effects of suspended nanoparticles on three dimensional MHD flow, heat and mass transfer of radiating Eyring - Powell fluid over a stretching sheet. J. Nanofluids 2015; 4:474.

21. Uddin MJ, Rana P, Bég O. Anwar et al. Finite element simulation of magnetohydrodynamic convective nanofluid slip flow in porous media with nonlinear radiation. Alex. Eng. J 2016; 55(2): 1305-1319.

22. Sheikholeslami M, Gorji-Bandpy M, Ganji DD and et al. Magnetohydrodynamic free convection of Al2O3-water nanofluid considering thermophoresis and Brownian motion effects. Computers \& Fluids 2014; 94: 147-160. 
23. Uddin MJ, Bég O. Anwar and Amin NS. Hydromagnetic transport phenomena from a stretching or shrinking nonlinear nanomaterial sheet with Navier slip and convective heating: a model for bio-nano-materials processing. J. Magnetism Magnetic Materials 2014; 368: 252-261.

24. Fiorani D. Surface Effects in Magnetic Nanoparticles, Nanostructure Science and Technology Series, Springer, Germany (2005).

25. Bég O. Anwar, Zueco J and Chang TB. Numerical analysis of hydromagnetic gravitydriven thin film micropolar flow along an inclined plane. Chemical Engineering Communications 2010; 198(3): 312- 331.

26. Ramadan HM and Chamkha AJ. Analytical solutions for hydromagnetic free convection of a particulate suspension from an inclined plate with heat absorption. Int. J. Fluid Mechanics Research 2000; 27: 447-467.

27. Subba Rao A, Prasad VR, Nagendra N, Bhaskar Reddy N and Beg OA, Non-similar computational solution for boundary layer flows of non-Newtonian fluid from an inclined plate with thermal slip. J. Applied Fluid Mechanics 2016; 9 (2): 795-807.

28. Usha $\mathrm{R}$ and Uma B. Long waves on a viscoelastic film flow down a wavy incline. Int. J. Non-Linear Mechanics 2004; 39: 1589-1602.

29. Bég O. Anwar, Ghosh SK, Narahari M, and Beg TA. Mathematical modelling of thermal radiation effects on transient gravity-driven optically-thick gray convection flow along an inclined plate with pressure gradient. Chemical Engineering Communications 2011; 198: $1-15$.

30. Sui J, Zheng L, Zhang X and Chen G. Mixed convection heat transfer in power law fluids over a moving conveyor along an inclined plate. Int. J. Heat and Mass Transfer 2015; 85: 1023-1033.

31. Noor NFM, Abbasbandy S and Hashim. Heat and mass transfer of thermophoretic MHD flow over an inclined radiate isothermal permeable surface in the presence of heat source/sink. Int. J. Heat and Mass Transfer 2012; 55: 2122- 2128.

32. Rana P, Bhargava $\mathrm{R}$ and Bég $\mathrm{O}$. Anwar. Numerical solution for mixed convection boundary layer flow of a nanofluid along an inclined plate embedded in a porous medium. Comp. Math. Appl.2012; 64: 2816-2832.

33. Abed AS. Wave convection regimes in an inclined layer of nanofluid. Int. J. Current Engineering and Technology 2014; 4: 464-468.

34. Rana P and Bég O. Anwar, Mixed convection flow along an inclined permeable plate: effect of magnetic field, nanolayer conductivity and nanoparticle diameter. Applied Nanoscience 2015; 5: 569-581.

35. Dhanai R, Rana $\mathrm{p}$ and Kumar L. MHD mixed convection nanofluid flow and heat transfer over an inclined cylinder due to velocity and thermal slip effects: Buongiorno's model. Powder Technology 2016; 288: 140-150.

36. Gorla RSR and Chamkha AJ. Natural convective boundary layer flow over a horizontal plate embedded in a porous medium saturated with a nanofluid. J. Modern Physics 2011; 2: 62-71.

37. Rana P, Bhargava R and Bég O. Anwar, Finite element simulation of unsteady MHD transport phenomena on a stretching sheet in a rotating nanofluid. Proc. IMECHE-Part N; J. Nanoengineering and Nanosystems 2013; 227: 77-99.

38. Chamkha AJ and Aly AM. MHD free convection flow of a nanofluid past a vertical plate in the presence of heat generation or absorption effects. Chem. Eng. Comm. 2011; 198: 425-441.

39. Bég O. Anwar, Khan MS, Karim I, Alam MM and Ferdows M. Explicit numerical study of unsteady hydromagnetic mixed convective nanofluid flow from an exponentially stretching sheet in porous media. Applied Nanoscience 2014; 4(8): 943-957. 
40. Chamkha AJ, Abdul R and Khaled A. Similarity solutions for hydromagnetic simultaneous heat and mass transfer by natural convection from an inclined plate with internal heat generation or absorption. Heat and Mass Transfer 2001; 37: 117-123.

41. Basu S. Near-Field Radiative Heat Transfer across Nanometer Vacuum Gaps, Elsevier, USA 2016.

42. Bég O. Anwar, Zueco J, Takhar HS, Bég T. A. and Sajid A. Transient non-linear optically-thick radiative-convective double-diffusive boundary layers in a Darcian porous medium adjacent to an impulsively started surface: network simulation solutions, Comm. Nonlinear Science and Numerical Simulation 2009; 14: 3856-3866.

43. Liron $\mathrm{N}$ and Wilhelm HE. Integration of the magnetohydrodynamic boundary layer equation by Meksyn's method. J. Appl. Math. Mech. (ZAMM) 1974; 54: 27-37.

44. Hamilton RL and Crosser OK. Thermal conductivity of heterogeneous two component system. Ind. Eng. Chem. Fundam 1962; 1: 187-191.

45. Kakac S, Pramuanjaroenkij A. Review of convective heat transfer enhancement with nanofluids. Int. J. Heat and Mass Transfer 2009; 52: 3187-3196.

46. Oztop HF and Abu-Nada E. Numerical study of natural convection in partially heated rectangular enclosures filled with nanofluids. Int. J. Heat Mass Transfer 2008; 29: 13261336.

47. Brinkman HC. The viscosity of concentrated suspensions and solution. J. Chem. Phys. 1952; 20: 571-581.

48. Vajravelu K and Rivera J. Hydromagnetic flow at an oscillating plate. Int. J. Non-Linear Mechanics 2003; 38: 305-312.

49. Das S, Jana M, Guria M and Jana RN. Unsteady viscous incompressible flow due to an oscillating plate in a rotating fluid. Int. J. Physical Sciences 2008; 12: 51-64.

50. Rajesh V, Debnath L and Sridevi Ch. Unsteady MHD free convection flow of nanofluid past an accelerated vertical plate with variable temperature and thermal radiation. Int. $J$. Comp. Appl. Math. DOI: 10.1007/s40819-016-0176-5, 2016.

51. Brewster MQ, Thermal Radiative Transfer and Properties, Wiley, New York 1992.

52. Reddy JN. An Introduction to the Finite Element Method, $3^{\text {rd }}$ ed. McGraw-Hill Book Company: New York, 2006.

53. Rajesh V, Bég O. Anwar and Mallesh MP. Transient nanofluid flow and heat transfer from a moving vertical cylinder in the presence of thermal radiation: Numerical study, Proc. IMECHE- Part N: J. Nanoengineering and Nanosystems 2014;

DOI: $10.1177 / 1740349914548712$

54. Narahari $\mathrm{M}$ and Ishak A. Radiation effects on free convection flow near a moving vertical plate with Newtonian heating. J. Appl. Sciences 2011; 11(7): 1096-1104.

55. Ciupiński L, Siemiaszko D, Rosiński M, Michalski A and Kurzydłowski KJ, Heat sink materials processing by pulse plasma sintering. Advanced Materials Research 2009; 59: $120-124$.

56. Guo $\mathrm{Y}, \mathrm{Wu} \mathrm{D}, \mathrm{Ma} \mathrm{G}$ and Guo D. Trailing heat sink effects on residual stress and distortion of pulsed laser welded Hastelloy C-276 thin sheets. J. Materials Processing Technology 2014; 214: 2891-2899.

57. Doumanidis C and Fourligkas N. Distributed-parameter control of the heat source trajectory in thermal materials processing. ASME J. Manuf. Sci. Eng 1996; 118(4); 571578.

58. Uddin MJ, Yusoff NHM, Bég O. Anwar and Ismail AI. Lie group analysis and numerical solutions for non-Newtonian nanofluid flow in a porous medium with internal heat generation. Physica Scripta 2013; 87: 1-14. 
59. Babayekhorasani F, Dunstan DE, Krishnamoorti R and Conrad JC. Nanoparticle dispersion in disordered porous media with and without polymer additives. Soft Matter 2016; 12: 5676-5683.

60. Rana P, Bhargava R, Bég O. Anwar and Kadir A. Finite element analysis of viscoelastic nanofluid flow with energy dissipation and internal heat source/sink effects, Int. J. Applied Computational Mathematics (USA) (2016). DOI 10.1007/s40819-016-0184-5 (27 pages) 